\title{
Identification, Analysis and Gene Cloning of the SWEET Gene Family Provide Insights into Sugar Transport in Pomegranate (Punica granatum)
}

\author{
Xinhui Zhang 1,2 1 , Sha Wang ${ }^{1,2}$, Yuan Ren ${ }^{1,2}$, Chengyan Gan ${ }^{1,2}$, Bianbian Li 1,2, Yaoyuwei Fan ${ }^{1,2}$, \\ Xueqing Zhao ${ }^{1,2}$ (D) and Zhaohe Yuan ${ }^{1,2, *}$ \\ 1 Co-Innovation Center for Sustainable Forestry in Southern China, Nanjing Forestry University, \\ Nanjing 210037, China; zhxinhui@njfu.edu.cn (X.Z.); wangsha@njfu.edu.cn (S.W.); \\ renyuan426@njfu.edu.cn (Y.R.); gcy@njfu.edu.cn (C.G.); libianbian@njfu.edu.cn (B.L.); \\ fyyw@njfu.edu.cn (Y.F.); zhaoxq402@163.com (X.Z.) \\ 2 College of Forestry, Nanjing Forestry University, Nanjing 210037, China \\ * Correspondence: zhyuan88@hotmail.com
}

Citation: Zhang, X.; Wang, S.; Ren, Y.; Gan, C.; Li, B.; Fan, Y.; Zhao, X.; Yuan $Z$. Identification, Analysis and Gene Cloning of the SWEET Gene Family Provide Insights into Sugar Transport in Pomegranate (Punica granatum). Int. J. Mol. Sci. 2022, 23, 2471. https://doi.org/10.3390/ijms23052471

Academic Editor: Scott D. Russell

Received: 29 January 2022

Accepted: 21 February 2022

Published: 23 February 2022

Publisher's Note: MDPI stays neutral with regard to jurisdictional claims in published maps and institutional affiliations.

Copyright: (C) 2022 by the authors. Licensee MDPI, Basel, Switzerland. This article is an open access article distributed under the terms and conditions of the Creative Commons Attribution (CC BY) license (https:// creativecommons.org/licenses/by/ $4.0 /)$.

\begin{abstract}
Members of the sugars will eventually be exported transporter (SWEET) family regulate the transport of different sugars through the cell membrane and control the distribution of sugars inside and outside the cell. The SWEET gene family also plays important roles in plant growth and development and physiological processes. So far, there are no reports on the SWEET family in pomegranate. Meanwhile, pomegranate is rich in sugar, and three published pomegranate genome sequences provide resources for the study of the SWEET gene family. 20 PgSWEETs from pomegranate and the known Arabidopsis and grape SWEETs were divided into four clades (I, II, III and IV) according to the phylogenetic relationships. PgSWEETs of the same clade share similar gene structures, predicting their similar biological functions. RNA-Seq data suggested that PgSWEET genes have a tissue-specific expression pattern. Foliar application of tripotassium phosphate significantly increased the total soluble sugar content of pomegranate fruits and leaves and significantly affected the expression levels of PgSWEETs. The plant growth hormone regulator assay also significantly affected the PgSWEETs expression both in buds of bisexual and functional male flowers. Among them, we selected PgSWEET17a as a candidate gene that plays a role in fructose transport in leaves. The $798 \mathrm{bp}$ CDS sequence of $P g S W E E T 17 a$ was cloned, which encodes 265 amino acids. The subcellular localization of PgSWEET17a showed that it was localized to the cell membrane, indicating its involvement in sugar transport. Transient expression results showed that tobacco fructose content was significantly increased with the up-regulation of $\operatorname{PgSWEET17a}$, while both sucrose and glucose contents were significantly down-regulated. The integration of the PgSWEET phylogenetic tree, gene structure and RNA-Seq data provide a genome-wide trait and expression pattern. Our findings suggest that tripotassium phosphate and plant exogenous hormone treatments could alter PgSWEET expression patterns. These provide a reference for further functional verification and sugar metabolism pathway regulation of PgSWEETs.
\end{abstract}

Keywords: pomegranate; SWEET gene family; gene expression; gene cloning; subcellular localization

\section{Introduction}

Sucrose can be degraded to hexose (glucose and fructose), which is the main carbon source for plant growth and development or can act as osmoregulation substances and signal molecules. Especially in fruit crops, the accumulation of soluble sugars is not only a source of energy for fruit growth, but also determines to some extent the quality of the fruit [1]. Notably, sugar accumulation requires sugar metabolism and transport, in which sugar transporters play an integral role [2,3]. It is well known that the carbohydrate partitioning process is achieved from source to sink tissues via the element/companion cell 
(SE/CC) complex of the phloem [3,4]. In the leaves of many herbaceous plants, including most crops, the SE/CC complex shares very little of the plasmodesmata with adjacent cells. Thus, sucrose transport in mesophyll cells is exported to the cell wall probably by sugars will eventually be exported transporters (SWEETs), and then through sucrose transporters (SUTs/SUCs) into the SE/CC complex [4].

Currently, although the SWEET gene family has not been studied for a long time, the available findings suggest that they are involved in important physiological processes of plant growth and development (e.g., plant nectar production, seed and pollen development) by regulating the transport, distribution and storage of carbohydrate compounds $[5,6]$. SWEET proteins utilize intracellular and extracellular sugar concentration gradients for transmembrane movement, not proton gradients, so their sugar transport activity is not affected by environmental $\mathrm{pH}$. More importantly, SWEET proteins can perform bidirectional sugar transport across the membrane driven by solute potential along concentration gradients [7,8]. Other known monosaccharide transporters (MSTs) and SUTs are coupled to $\mathrm{H}^{+}$and perform unidirectional transmembrane sugar transport through intracellular and extracellular $\mathrm{H}^{+}$concentration gradients $[9,10]$. Unlike SUTs and MSTs, which contain $12 \alpha$-helical transmembrane (TM) structural domains connected by hydrophilic loops, SWEET genes belonging to the MtN3/saliva family encode membrane proteins that typically have seven conserved TM domains linked by a PQ-loop-repeat [11]. The topology of SWEETs is different from that of SUTs and MSTs, which may be important for the different sugar transport.

In plants, phylogenetic analysis has shown that SWEETs can be divided into four clades (I, II, III and IV), and genes in the same subclade have similar gene structures and functions [12,13]. Much evidence suggests that Clades I and II may transport glucose, Clade III prefers sucrose, and Clade IV is an efficient fructose transporter. SWEETs act as sugar transporters involved in phloem loading during the process of sucrose metabolism (Table 1). Chen et al. [7] first demonstrated that AtSWEET11/12 are located in phloem parenchyma, are highly expressed, and can transport sucrose from leaves to vascular bundles. AtSWEET17 is highly expressed in the vacuolar membrane in Arabidopsis leaves and root and is responsible for fructose bidirectional transport [14]. Members of the SWEET family are frequently involved in plant reproduction and development (Table 1). AtSWEET9 is specifically expressed in parenchyma cells of nectary, while mutant atsweet 9 results in reduced nectar production [15]. Ossweet14 mutant knockouts caused less plump seeds and delayed growth, suggesting that OsSWEET14 plays a role in grain filling [16]. Meanwhile, OsSWEET1a, OsSWEET2a, OsSWEET3a, OsSWEET4, OsSWEET5 and OsSWEET15 were highly expressed in plant flowers and panicles, indicating that these transporters also play a certain role in rice reproductive development [17]. In addition, SWEETs are also involved in ion transport, leaf senescence, plant-pathogen interaction and abiotic stress [18-21]. 
Table 1. SWEET genes involved in physiological function.

\begin{tabular}{|c|c|c|c|c|c|c|}
\hline Clade & Gene & $\begin{array}{l}\text { Involved in Sugar } \\
\text { Transport }\end{array}$ & Reference & Gene & $\begin{array}{c}\text { Involved in Reproductive } \\
\text { Development }\end{array}$ & Reference \\
\hline \multirow[t]{2}{*}{ I } & AtSWEET1 & plasma membrane & [8] & AtSWEET1 & petal & \\
\hline & & & & OsSWEET $2 a$ & flower and panicle & [22] \\
\hline \multirow[t]{6}{*}{ II } & & & & $\mathrm{ZmSWEET4c}$ & basal endosperm transfer layer & {$[23]$} \\
\hline & & & & AtSWEET5 & mature pollen grain & {$[24]$} \\
\hline & & & & LeSWEET5b & mature pollen grain & {$[25]$} \\
\hline & & & & OsSWEET5 & $\begin{array}{l}\text { during flower and panicle } \\
\text { development }\end{array}$ & [22] \\
\hline & & & & AtSWEET7 & during pollen development & [26] \\
\hline & & & & AtSWEET8 & $\begin{array}{l}\text { during pollen wall and anther } \\
\text { development }\end{array}$ & [27] \\
\hline \multirow[t]{5}{*}{ III } & AtSWEET11 & phloem cell & [7] & AtSWEET9 & nectary parenchyma cell & [15] \\
\hline & AtSEET12 & phloem cell & [7] & NaSWEET9 & nectary cell & {$[15]$} \\
\hline & OsSWEET11 & phloem cell & [7] & AtSWEET13 & stamen & [28] \\
\hline & OsSWEET14 & phloem cell & [7] & AtSWEET14 & stamen & {$[16]$} \\
\hline & OsSWEET15 & senescent leaf & [21] & OsSWEET11 & panicle and anther & [29] \\
\hline \multirow[t]{2}{*}{ IV } & AtSWEET16 & root & [30] & & & \\
\hline & AtSWEET17 & leaf and root & [20] & & & \\
\hline
\end{tabular}

Note: At represents Arabidopsis, Os represents rice, Le represents tomato, $\mathrm{Zm}$ represents maize.

Pomegranates are grown in different subtropical and tropical microclimatic zones around the world, such as Iran, California, Turkey, Egypt, Italy, India, Chile, Spain, and China. The market demand for pomegranate products such as pomegranate and its by-products juice, jam, and pomegranate wine are steadily increasing due to the growing consumer interest in the potential benefits of pomegranate and its phytochemicals. Pomegranate has been used since ancient times for the prevention and treatment of several diseases, has strong antioxidant activity, and is rich in anthocyanins, tannins and unique punicalagin. Studies have shown that pomegranate fruit and its juice, extracts and oils are able to exert anti-inflammatory, anti-proliferative and anti-tumor effects by modulating multiple signaling pathways [31-34].

At present, studies on pomegranate sugar metabolism have so far focused on the determination of soluble sugar components and contents, and there is a lack of research on the regulatory mechanism of sugar metabolism. Pomegranate juice is classified into three categories based on its soluble solids (TSS) and titratable acid (TA) content; juice with TSS above $13 \%$ and TA below $0.7 \%$ is classified as sweet pomegranate, juice with TSS range of $12-13 \%$ and TA of $0.7-1.8 \%$ is classified as sweet and sour pomegranate, and juice with TSS below $12 \%$ and TA above $1.8 \%$ is classified as sour pomegranate [35]. In addition, the flower buds of pomegranate are distinguished as fertile and abortive flowers. Flower development consists of two stages including (i) the transition from vegetative to reproductive development and (ii) organogenesis, both of which are regulated by a number of well-characterized key genes leading to abortive flowers [36]. Interestingly, it has been found that sugar/hormone interactions, or direct effects on the transcription of genes that regulate flower induction, ensure flower bud formation [37-39].

Studying the function and regulatory mechanism of the pomegranate SWEET gene family can help regulate the pattern of sugar metabolism as well as fruit, leaf and flower development. The completion of pomegranate whole genome sequencing and the publication of data provide important data to support the study of pomegranate gene function [40-42]. Using bioinformatics methods, we identified 20 PgSWEETs, and analyzed their physical and chemical properties, phylogenetic relationships, gene structures and RNA-seq data. We also explored the expression patterns of PgSWEETs after foliar application of tripotassium phosphate and plant growth hormone regulators. Finally, gene cloning, subcellular localization and transient expression of tobacco of PgSWEET17a were analyzed to provide reference for studying its biological functions in pomegranate sugar transport. 


\section{Results}

2.1. PgSWEET Gene Family Members Identification and Sequence Analysis

We identified 20 pomegranate SWEETs according to the Pfam database (ID: PF03083). Physicochemical properties of the genes were analyzed through online software Protparam (Table 2). Analysis showed that length of amino acid of 20 PgSWEETs were ranged from 213 (PgSWEET7a) to 448 (PgSWEET1e) aa. Molecular weight was from 23,370.82 (PgSWEET17b) to 50,147.21 (PgSWEET1e) ku. The pI of 2 PgSWEETs (PgSWEET17b and PgSWEET12) were less than 7 , which were more acidic, while others were more than 7 , which were more basic.

Table 2. Basic information of the SWEET gene family in pomegranate.

\begin{tabular}{|c|c|c|c|c|c|c|}
\hline Gene Name & Gene ID & Location & Exon No. & $\begin{array}{c}\text { Protein } \\
\text { Length/aa }\end{array}$ & $\begin{array}{l}\text { Molecular } \\
\text { Weight/ku }\end{array}$ & pI \\
\hline PgSWEET10 & Pg000003.1 & scaffold1:6849801;6852242 & 6 & 318 & $35,423.91$ & 8.46 \\
\hline PgSWEET5 & Pg000836.1 & scaffold1:5975742;5977070 & 6 & 240 & $27,038.52$ & 9.73 \\
\hline PgSWEET15 & Pg002682.1 & scaffold11:951702;953916 & 6 & 304 & $34,149.26$ & 7.65 \\
\hline PgSWEET2 & Pg006257.1 & scaffold14:1751672;1753913 & 6 & 235 & $26,053.98$ & 9.12 \\
\hline PgSWEET16a & Pg007971.1 & scaffold16:3103840;3105566 & 6 & 311 & $34,217.96$ & 9.10 \\
\hline PgSWEET16b & Pg007972.1 & scaffold16:3100513;3102712 & 6 & 314 & $34,865.04$ & 9.29 \\
\hline PgSWEET17b & Pg007973.1 & scaffold16:3089934;3093052 & 5 & 215 & $23,370.82$ & 6.09 \\
\hline PgSWEET1a & Pg008613.1 & scaffold165:311392;314797 & 5 & 253 & $28,013.80$ & 9.33 \\
\hline PgSWEET1d & Pg008635.1 & scaffold165:171819;177396 & 6 & 242 & $27,050.67$ & 9.30 \\
\hline PgSWEET1C & Pg008637.1 & scaffold165:213966;219285 & 6 & 259 & $28,898.66$ & 9.25 \\
\hline PgSWEET17a & Pg012205.1 & scaffold21:467086;471865 & 6 & 250 & $27,850.58$ & 7.79 \\
\hline PgSWEET8 & Pg015006.1 & scaffold26:1968903;1972285 & 5 & 239 & $26,816.01$ & 9.29 \\
\hline PgSWEET1b & Pg015079.1 & scaffold260:29495;32112 & 5 & 227 & $25,323.43$ & 9.18 \\
\hline PgSWEET9 & $\operatorname{Pg} 017691.1$ & scaffold33:1183976;1186154 & 6 & 270 & $30,135.24$ & 9.52 \\
\hline PgSWEET1e & Pg019984.1 & scaffold4:1483201;1490504 & 7 & 448 & $50,147.21$ & 9.61 \\
\hline PgSWEET3 & Pg020538.1 & scaffold42:1971076;1972515 & 6 & 257 & $28,524.69$ & 9.25 \\
\hline PgSWEET12 & Pg027298.1 & scaffold72:134285;136352 & 6 & 325 & $35,756.12$ & 6.16 \\
\hline PgSWEET7a & Pg029959.1 & scaffold9:1816650;1819681 & 3 & 213 & $23,424.94$ & 9.25 \\
\hline PgSWEET7b & Pg029960.1 & scaffold9:1824680;1828883 & 5 & 258 & $28,304.74$ & 9.19 \\
\hline PgSWEET11 & Pg030654.1 & scaffold97:711886;713925 & 6 & 275 & $30,531.75$ & 9.33 \\
\hline
\end{tabular}

\subsection{Phylogenetic Tree Analysis}

We identified 20 PgSWEETs from pomegranate, then reconstructed one gene tree with known AtSWEETs and VvSWEETs for further study SWEET family evolutionary relationship (Figure 1). Apparently, SWEETs from three species were split into four clades (I, II, III and IV, Figure 1). Among them, Clade I has 7 PgSWEETs, Clade II has 4 PgSWEETs, Clade III has 5 PgSWEETs, and Clade IV has 4 PgSWEETs. There is a germline-specific clade (PgSWEET1a-1e) of pomegranate in Clade I. 


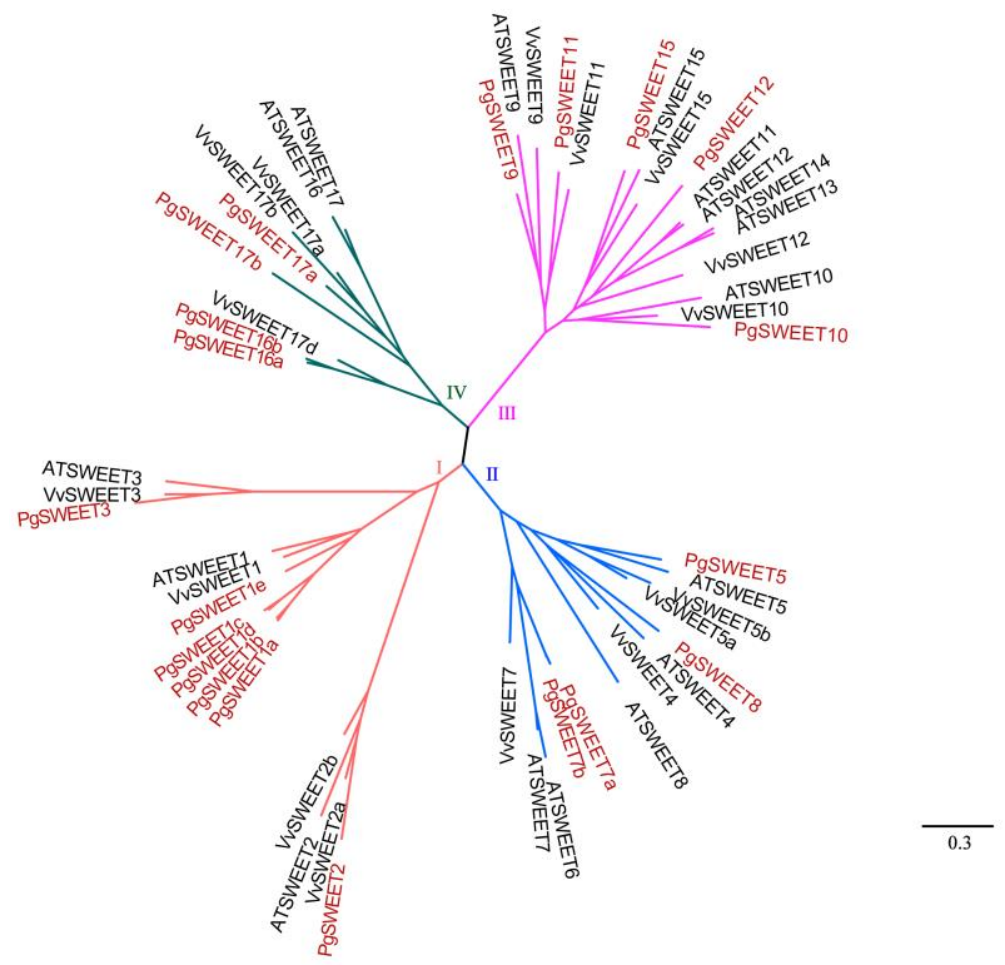

Figure 1. Phylogenetic tree of the SWEET gene family in pomegranate, Arabidopsis and grape.

\subsection{PgSWEETs Gene Structure and Phylogenetic Tree Analysis}

To investigate the phylogenetic relationships of the PgSWEET gene family, we reconstructed the evolutionary tree with 20 pomegranate gene sequences by IQ-tree (Figure 2A). Apparently, phylogenetic analysis showed robust support for Clades I, II and IV, and less support for Clade III. Five homologous pairs were formed among these 20 sequences, including PgSWEET1 $a$ and PgSWEET1b, PgSWEET1c and PgSWEET1d, PgSWEET7c and $P g S W E E T 7 d, P g S W E E T 16 a$ and PgSWEET16b, and PgSWEET17a and PgSWEET17b. All pairs, except $P g S W E E T 17 a$ and $P g S W E E T 17 b$, possessed an approval rate of 100, indicating a very strong phylogeny and a close association with each other.
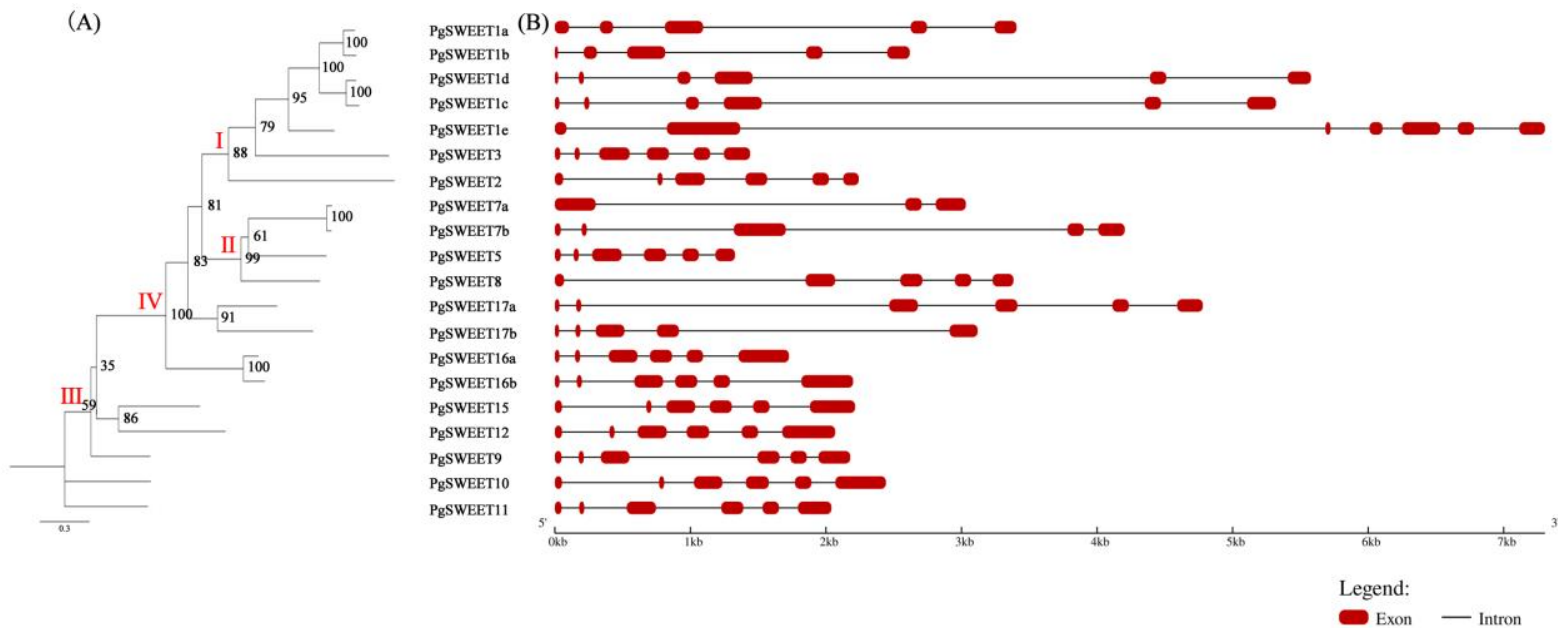

Figure 2. Phylogenetic tree (A) and gene structures (B) of the pomegranate SWEET gene family.

We utilized the online software GSDS for investigating the PgSWEET gene structural features (Figure 2B). Structural analysis showed that most genes have 5 or 6 exons, while $P g S W E E T 7 a$ only has 3 exons and PgSWEET1e has 7 exons. The most obvious difference 
among these genes structure is the length of introns. Genes in Clade I have the longest introns, and those in Clade III have shorter introns. The length of introns grows gradually as the phylogenetic tree branches from the outer to the inner branches. Combined with phylogenetic tree and Scaffold location analysis, PgSWEET1b and PgSWEET1c, PgSWEET7a and $P g S W E E T 7 b$, and $P g S W E E T 16 a$ and $P g S W E E T 16 b$ were found to have tandem duplication.

\subsection{PgSWEETs Gene Expression Analysis}

To further investigate the gene expression divergence among different tissues, we downloaded RNA-seq data from NCBI for pomegranate (Table 3, Figure 3). Among the 20 PgSWEET gene expressions in different tissues, five genes (PgSWEET1a, 1b, 3, 8 and $17 b$ ) were not expressed or minimally expressed in various tissues. PgSWEET1d was highly expressed only in the root of 'Dabenzi'. Four genes (PgSWEET5, 7a, 9 and 11) showed similar expression patterns, and in the flower of 'Dabenzi', mixed samples of 'Black127' and 'Nana' showed higher expression levels. In particularly, PgSWEET7a and 11 showed the highest transcript levels in the flower of 'Dabenzi'. PgSWEET1c and $1 d$ displayed the highest expression levels in the root. Only three genes PgSWEET2, 15 and 16b displayed expression in all tissues. Among them, PgSWEET2 expression in all tissues were all higher, indicating that $P g S W E E T 2$ may play a multifaceted function in the growth and development of pomegranate.

Table 3. RNA-seq data of pomegranate.

\begin{tabular}{|c|c|c|c|c|}
\hline Accession No. & Cultivar & Sample & ID & Reference \\
\hline SRR1054190 & Black 127 & Mixed samples of root, leaf, flower and fruit & Black 127 & [43] \\
\hline SRR1055290 & nana & Mixed samples of root, leaf, flower and fruit & nana & [43] \\
\hline SRR5279388 & Dabenzi & Outer seed coat & DBZ_OSC & [42] \\
\hline SRR5279391 & Dbenzi & Inner seed coat & DBZ_ISC & [42] \\
\hline SRR5279394 & Dabenzi & Pericarp & DBZ_pericarp & [42] \\
\hline SRR5279395 & Dabenzi & Flower & DBZ_flower & [42] \\
\hline SRR5279396 & Dabenzi & Root & DBZ_root & [42] \\
\hline SRR5279397 & Dabenzi & Leaf & DBZ_leaf & [42] \\
\hline SRR5446598 & Tunisia & $3-5 \mathrm{~mm}$ bud of bisexual flower & $3-5 \mathrm{~mm}(\mathrm{~B})$ & [44] \\
\hline SRR5446595 & Tunisia & $5.1-13 \mathrm{~mm}$ bud of bisexual flower & $5.1-13 \mathrm{~mm}(\mathrm{~B})$ & [44] \\
\hline SRR5446592 & Tunisia & 13.1-25 mm bud of bisexual flower & $13.1-25 \mathrm{~mm}(\mathrm{~B})$ & {$[44]$} \\
\hline SRR5446607 & Tunisia & $3-5 \mathrm{~mm}$ bud of functional male flower & 3-5 mm (F) & [44] \\
\hline SRR5446604 & Tunisia & $5.1-13 \mathrm{~mm}$ bud of functional male flower & $5.1-13 \mathrm{~mm}(\mathrm{~F})$ & {$[44]$} \\
\hline SRR5446601 & Tunisia & $13.1-25 \mathrm{~mm}$ bud of functional male flower & $13.1-25 \mathrm{~mm}(\mathrm{~F})$ & [44] \\
\hline
\end{tabular}

2.5. Changes in Soluble Sugar Concentration and PgSWEETs Expression Pattern of Pomegranate Fruit and Leaf after Tripotassium Phosphate Treatment

When the pomegranate fruit enters the color turning period (4-5 weeks before harvest), the growth of the fruit and the accumulation of sugar require nutrients, and the demand for fertilizer increases at this stage, which is the most demanding stage in the whole growth process. At this time, in addition to basic and reasonable fertilization, proper foliar application can supplement sufficient nutrients to improve fruit growth, increase sweetness, and enable successful coloring. Therefore, we started foliar spraying of "Big Seed" pomegranate with tripotassium phosphate solution from September 20 (pomegranate fruit color change phase) to 10 October. Compared with the control group, the results showed that fruit TSS/TA, fruit and leaf total soluble sugar contents were significantly enhanced after foliar spraying with tripotassium phosphate (Figure 4A-C). 


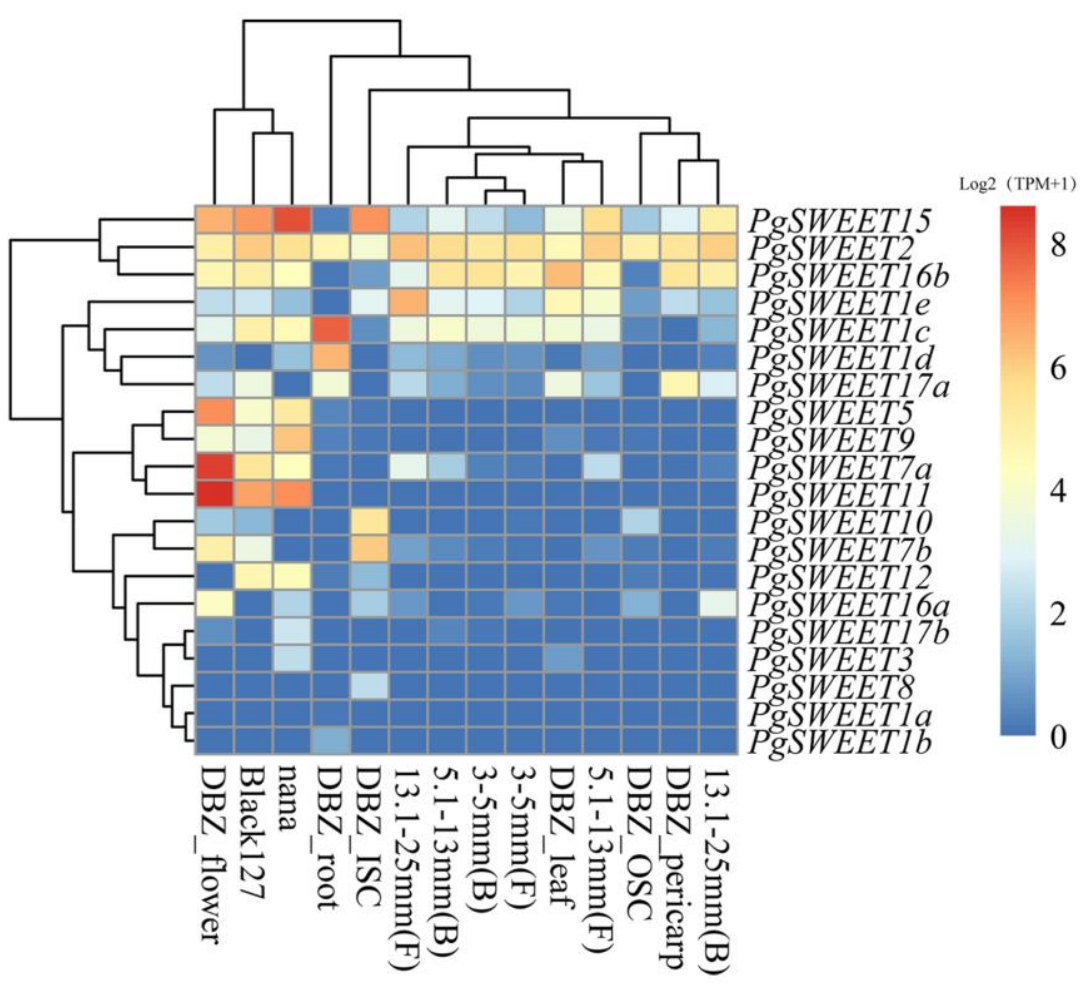

Figure 3. Expression heatmap of SWEET genes in different tissues of pomegranate.

(A)

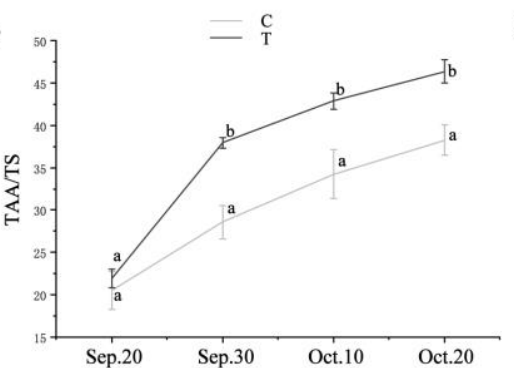

(B)

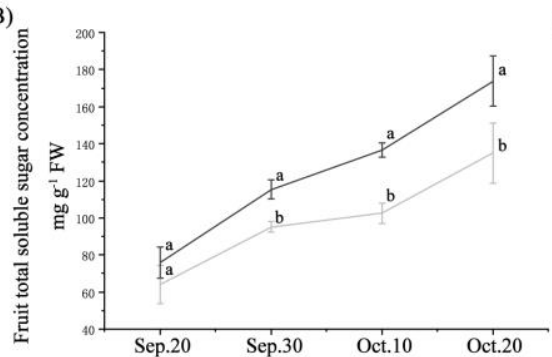

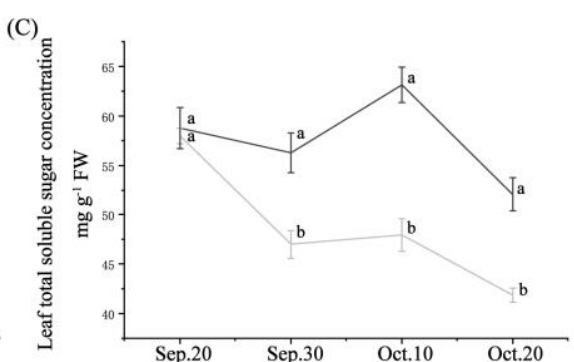

(E)
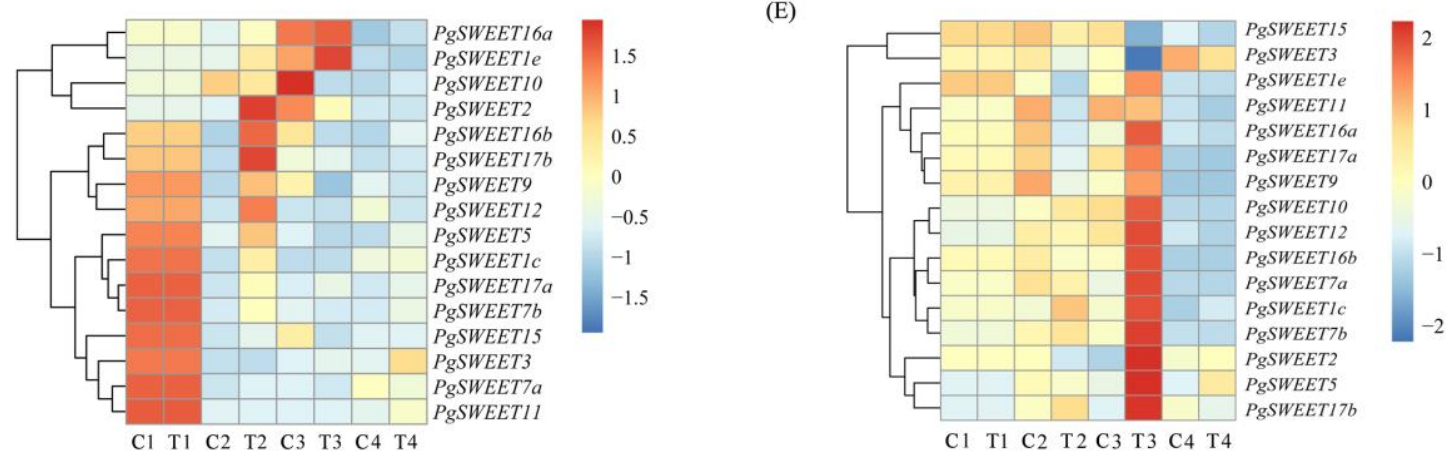

$\begin{array}{lllllllll}\mathrm{C} 1 & \mathrm{~T} 1 & \mathrm{C} 2 & \mathrm{~T} 2 & \mathrm{C} 3 & \mathrm{~T} 3 & \mathrm{C} 4 & \mathrm{~T} 4\end{array}$

Figure 4. Changes in TSS/TA of fruit (A), total soluble sugar concentration of fruit (B), total soluble sugar concentration of leaf (C), SWEETs expression of fruit (D) and SWEETs expression of leaf (E) during fruit growth and development after pure water (C, control) and tripotassium phosphate ( $\mathrm{T}$, treatment). Each value is the mean for three replicates, with vertical bars representing standard errors. The different letters at each time point show significant differences $(p<0.05)$ using Duncan's tests between control and treatment. In (D,E), numbers 1-4 represent dates from 20 September to 20 October 2020. 
To investigate the effect of foliar application of tripotassium phosphate on the fruit sugar transporter, the transcript levels of 16 PgSWEET genes were determined (Figure 4D). The expression of SWEETs in pomegranate fruits was generally low from the color turning stage to the ripening stage, and the expression of the same gene varied greatly at different times. Overall, the expression of SWEETs in fruits was relatively high on 20 September, and the expression of nine genes (PgSWEET1c, 3, 5, 7a, 7b, 9, 11, 15 and 17a) was the highest compared with other periods. Foliar application of tripotassium phosphate also altered the expression pattern of SWEETs in fruits, with the highest expression of PgSWEET2 and $16 b$ in the treatment group on 30 September and the highest expression of PgSWEET1e and $16 a$ in the treatment group on 10 October. In addition, foliar fertilization decreased the expression of $\mathrm{PgSWEET10}$ compared to the control group.

To explore the functional differences of sugar transport in source organs after foliar application of tripotassium phosphate compared with the control, we determined the expression of 16 PgSWEET family genes in the treated and control groups of leaves and drew a clustering heat map (Figure 4E). There were differences in the expression of PgSWEETs between different periods and treatments. It was clearly seen that the expression of PgSWEETs was significantly higher in the treatment group on 10 October, where the expression of 13 genes (PgSWEET1c, 1e, 2, 5, 7a, 7b, 9, 10, 12, 16a, 16b, 17a and 17b) was higher than in other stages and the expression of PgSWEET11 was also at a higher level. On 20 October, gene expressions were lower in both control and treated groups except for PgSWEET2, 3 and 5, and it is speculated that leaves started to enter the senescence stage. There were also significant differences in the expression of PgSWEETs between the treatment and control groups on 30 September. For example, after foliar application of tripotassium phosphate, the expression levels of $\mathrm{PgSWEET1c}$ and $17 \mathrm{~b}$ were significantly higher than those of the control group, while the expression levels of PgSWEET1e, 2, 9, 11, $16 a$ and $17 a$ were significantly lower than those of the control group.

\subsection{Changes in Buds Total Soluble Sugar Contents and PgSWEETs Expression Pattern of Bisexual Flower and Functional Male Flower after Hormone Treatment}

We measured the buds sugar content at different periods under hormone treatment and found that their sugar content was affected by hormones (Figure 5A,B). Then, the expression of some PgSWEETs was measured to explore their changes during this process (Figure 5).

The qPCR results showed that the expression of PgSWEET1c and PgSWEET2 in CK was higher in bisexual flowers than in functional male flowers. The expression of PgSWEET1e and PgSWEET15 was lower in bisexual flowers than in functional male flowers. The expression of $P g S W E E T 16 b$ was significantly higher in the P3 stage of bisexual flowers than in functional male flowers, and the expression of PgSWEET17a was lower in the P1 stage of bisexual flowers than in functional male flowers. The expression of PgSWEET17a was lower in the P1 stage of bisexual flowers than in functional male flowers. In the CK group of bisexual flowers, PgSWEET1c, 1e, 15 and 17a expression was highest in the P2 stage, and $P g S W E E T 2$ and $16 b$ were highest in the P3 stage. In functional male flowers of the CK group, PgSWEET1c, 1e, and 16b expression was highest in the P2 stage, PgSWEET2 and 17a expression continued to decrease, and PgSWEET15 continued to increase.

Under IBA treatment, PgSWEET1c and $16 b$ were down-regulated in both types of flowers compared to the control. PgSWEET1e, 2 and 15 were up-regulated in bisexual flowers due to exogenous IBA treatment and down-regulated in functional male flowers. $P g S W E E T 17 a$ was up-regulated in all three stages in bisexual flowers and up-regulated in functional male flowers at the P1 and P3 stages, whereas it was down-regulated at the P2 stage. 
(A)
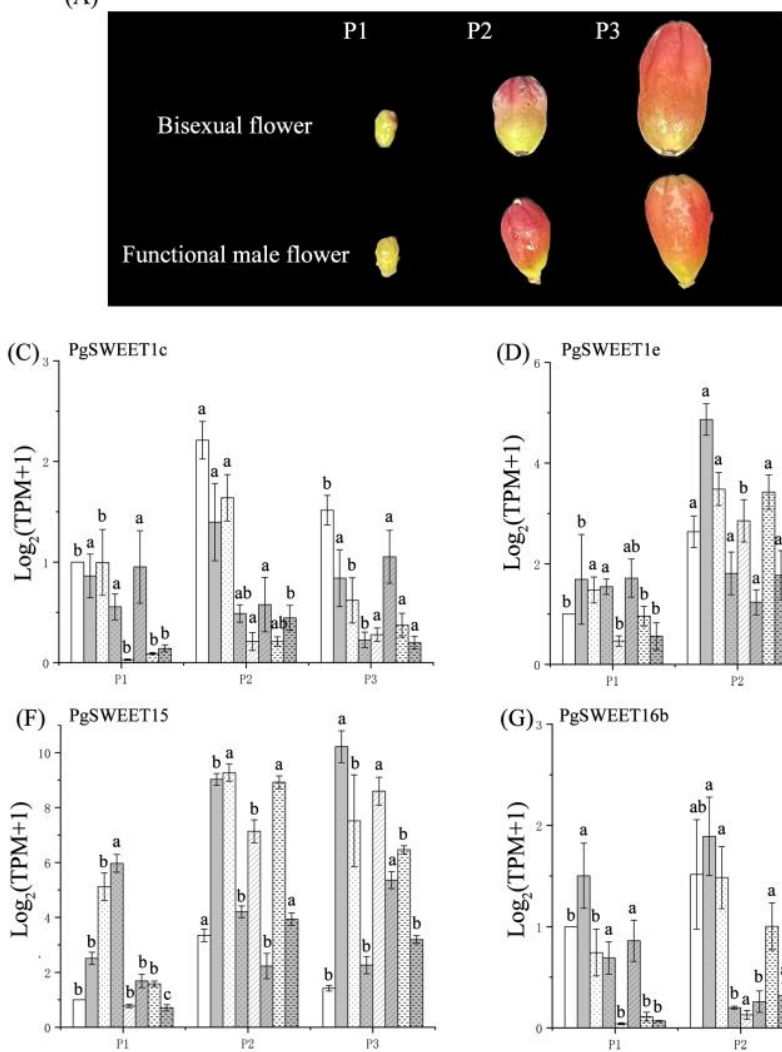

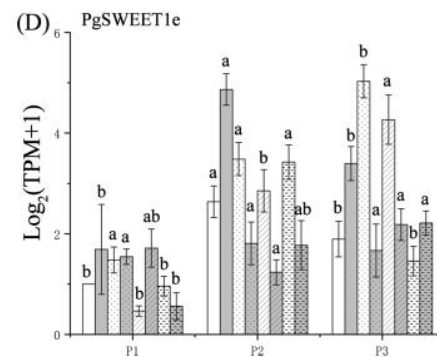

$(\mathrm{G})_{3}$ PgSWEET16b

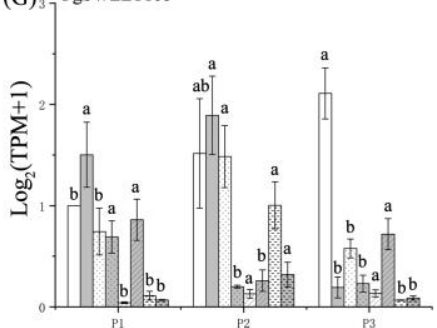

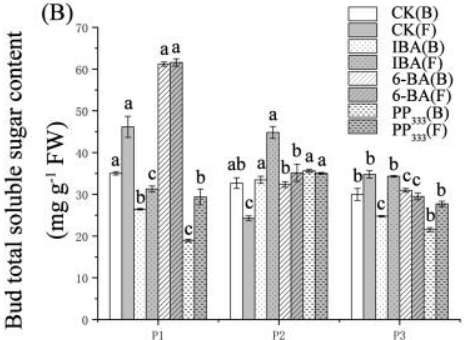
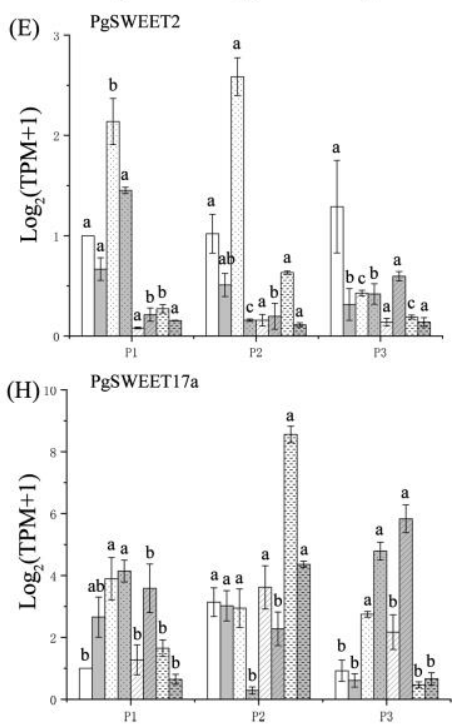

Figure 5. Buds of bisexual flower and functional male flowers during different stages (A). Changes in buds total soluble sugar content $(\mathbf{B})$ and SWEETs expression $(\mathbf{C}-\mathbf{H})$ of bisexual and functional male flowers under hormone treatment. Each value is the mean for three replicates, with vertical bars representing standard errors. The different letters at each time point show significant differences $(p<0.05)$ using Duncan's tests between P1, P2 and P3. In the note, B represents bisexual flower, F represents functional male flower.

Under 6-BA treatment, $P g S W E E T 1 c, 2$ and $16 b$ were down-regulated in expression at all three stages in bisexual flowers compared with control, whereas PgSWEET17a was up-regulated. PgSWEET1e and 15 expression were down-regulated at the P1 stage and up-regulated at the P2 and P3 stages in bisexual flowers. Although the expression of the six PgSWEETs in functional male flowers under 6-BA treatment was variable, it could be found that the expression of all six PgSWEETs was significantly lower than control at the P2 stage, a critical period for sterile flower abortion.

Under $\mathrm{PP}_{333}$ treatment, the expression of all six PgSWEETs was down-regulated in functional male flowers compared to control, except for PgSWEET17a, which was downregulated at the P1 and P3 stages. PgSWEETs expression changes were more complex in bisexual flowers compared with functional male flowers. In bisexual flowers, PgSWEET1c, 2 , and $16 b$ were all down-regulated, and PgSWEET15 was up-regulated. PgSWEET1e and $17 a$ were up-regulated at the $\mathrm{P} 1$ and $\mathrm{P} 2$ stages and down-regulated at the P3 stage compare with control.

Overall, under exogenous hormone treatment, PgSWEET1c, 2 and 16b expression may be down-regulated, and PgSWEET1e, 15 and $17 a$ expression was up-regulated in bisexual flowers compared with control. Six PgSWEETs were down-regulated in functional male flowers at all three stages or critical periods.

\subsection{Cloning and Structural Analysis of PgSWEET17a}

The AtSWEET17 protein sequence of Arabidopsis thaliana was utilized as the probe sequence, and the homologous gene sequence obtained was named PgSWEET17a by 
blastp comparison (http:/ / cucurbitgenomics.org/blast, accessed on 26 August 2021) in the pomegranate genome database. Amplification primers were designed according to the known CDS sequence, and PCR amplification was performed using cDNA mixes from pomegranate flower, leaf, fruit and skin tissues as templates, and the amplified products were analyzed by agarose gel electrophoresis, recovered, cloned and sequenced. The cDNA of the PgSWEET17a gene was obtained by cloning with a full length of $798 \mathrm{bp}$ and a CDS sequence of $798 \mathrm{bp}$ (Figure 6A) Pfam database search revealed that this protein has the $\mathrm{MtN} 3$ / saliva conserved structural domain (Figure 6B).
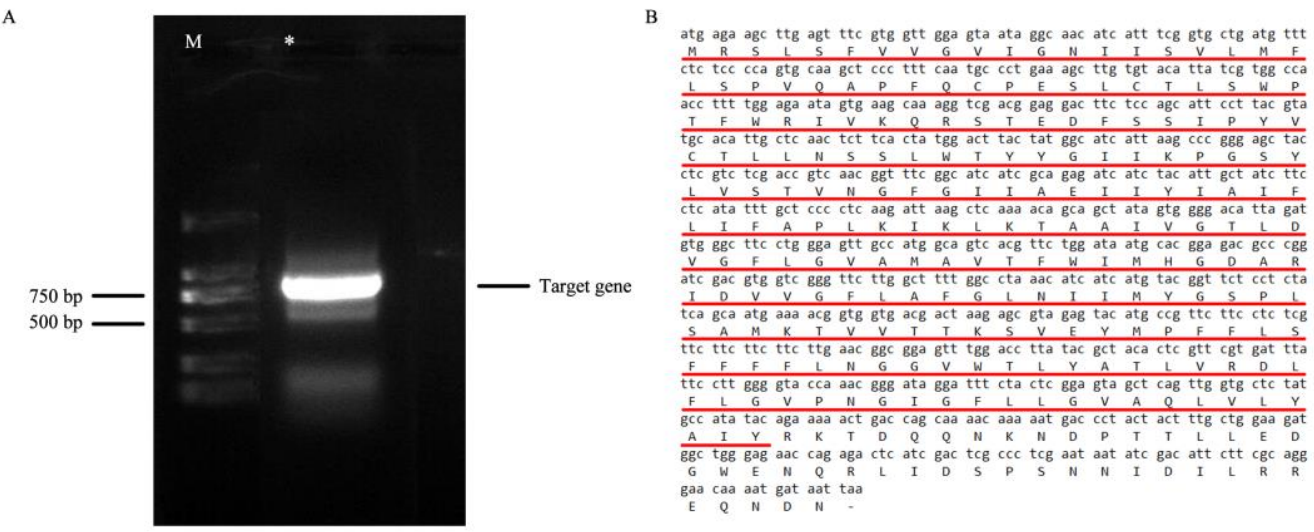

Figure 6. PCR amplification (A) and the coding sequence (B) of PgSWEET17a. Note: DL ladder 2000 DNA Marker; *: Pomegranate cDNA template; the red line is the MtN3/saliva domain.

Using physicochemical property analysis of the sequence, the results showed that PgSWEET17a protein encodes 265 amino acids and the molecular formula of the encoded product is $\mathrm{C} 1383 \mathrm{H} 2130 \mathrm{~N} 330 \mathrm{O} 369 \mathrm{~S} 10$ (Figure 6B), which is predicted by online software Plant-mPLoc to be localized on the cell membrane. It has a molecular weight of $29.60 \mathrm{kD}$, an aliphatic index of 112.87, a grand average of hydrophilicity of 0.532 , a theoretical isoelectric point (pI) of 6.57 , and an instability index of 44.44 .

\subsection{PgSWEET17a Conservative Domain Analysis}

Plant SWEET proteins belong to the MtN3/saliva family and generally contain seven TMs, of which TM4 is poorly conserved and acts as a linker, forming a 3-1-3 structure that forms two MtN3/saliva structural domains, each containing three TMs. In contrast, prokaryotes have only one MtN3/saliva structural domain, and it is speculated that eukaryotic SWEETs evolved through the internal replication (replication or horizontal gene transfer fusion) of the 3 TMs [8,45]. From Figure 7, PgSWEET17a apparently has 7 TMs.

To further study the conserved domain of PgSWEET17a MtN3/saliva, PgSWEET17a and PgSWEET17b from pomegranate and 13 SWEET proteins from grape, rice, tomato and Arabidopsis were aligned to detect conserved domains (Figure 7). PgSWEET17b is a homologous sequence of PgSWEET17a, while it has multiple base substitutions or deletions in conserved regions, which could result in neo- or sub-functionalization. The alignment exhibited that the residues G9, G12, N13, P23, T41, F42, P58, Y59, Y72, G73 and Y98 on THB1 and G149, P163 and L183 on THB2 were completely conserved. Besides, more than $90 \%$ of SWEETs have I45, T86, G89 and G91 on THB1 and S175, V176, P180, S184, Y225 and Y228 residues. Furthermore, the prolines (P) in TM1, TM2, TM5 and TM6 were conserved in 15 SWEETs, and only PgSWEET17b had a glutamine (Q)-substitution in the TM6, which may be responsible for the loss of activity in PgSWEET17b [46]. Similarly, two conserved residues N88 and N211 in TM3 and TM7, respectively, were vital for AtSWEET1 activity [46], and were reserved in 14 SWEETs except for PgSWEET17b. 




Figure 7. Sequence alignment of SWEET proteins.

\subsection{PgSWEET17a Subcellular Localization Analysis}

Predictive analysis of the subcellular localization of PgSWEET17a protein using the online tool Plant-mPLoc showed that it was localized to the cell membrane. To verify the above predicted results, the homologous recombinant PgSWEET17a-GFP fusion protein was transiently expressed in tobacco leaves, and the results showed that PgSWEET17a was localized to the cell membrane (Figure 8). It is presumed to be involved in sugar transport.

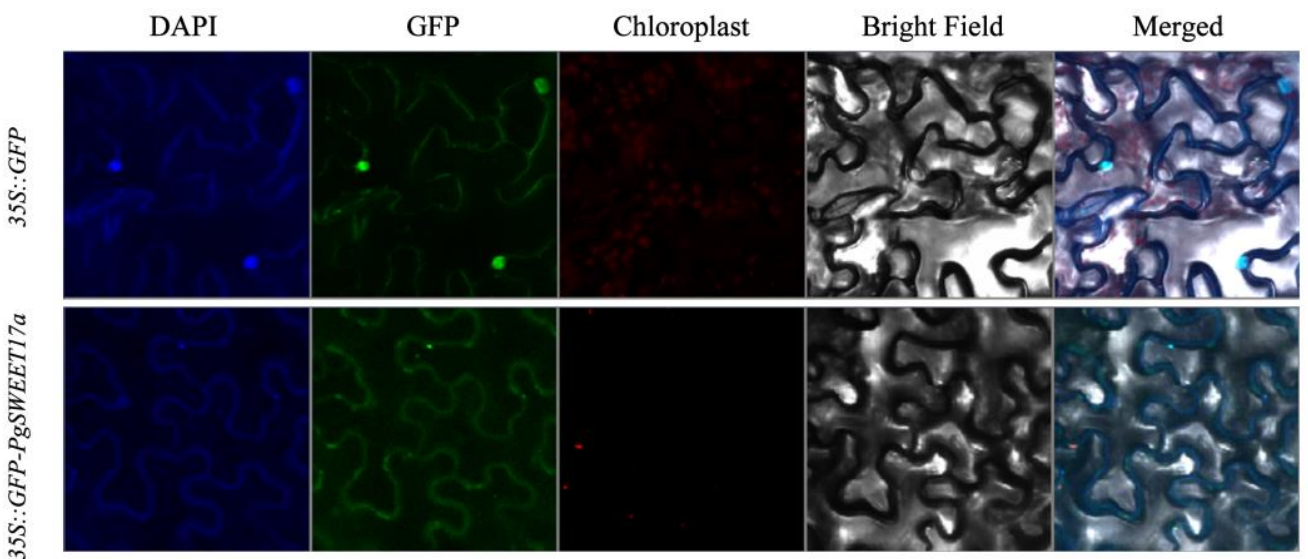

Figure 8. Subcellular localization of the 35S::GFP-PgSWEET17a fusion protein in tobacco leaves. Free GFP served as a control. A DAPI staining assay was conducted to confirm the nuclear localization.

\subsection{PgSWEET17a Cis-Acting Elements Analysis}

The results are shown in Table 4 , where the PgSWEET17a sequence contains cisacting elements associated with abiotic stresses and hormones. SREATMSD, ABRE, ARE, AuxRR-core, CGTCA-motif and CGTCA-motif are cis-acting elements associated with the sugar signal, abscisic acid responsiveness, anaerobic induction, auxin responsiveness, 
MeJA-responsiveness, light responsiveness, low-temperature responsiveness and droughtinducibility, respectively.

Table 4. Analysis of some important cis-acting regulatory elements in the promoter sequences of PgSWEET17a.

\begin{tabular}{ccc}
\hline Cis-Acting Element Name & Sequence & Function \\
\hline SREATMSD & TTATCC & Cis-acting element involved in the sugar signal \\
ABRE & ACGTG & Cis-acting element involved in the abscisic acid responsiveness \\
ARE & AAACCA & Cis-acting regulatory element essential for the anaerobic induction \\
AuxRR-core & GGTCCAT & Cis-acting regulatory element involved in auxin responsiveness \\
CGTCA-motif & CGTCA & Cis-acting regulatory element involved in the MeJA-responsiveness \\
G-Box & CACGTT & Cis-acting regulatory element involved in light responsiveness \\
LTR & CCGAAA & Cis-acting element involved in low-temperature responsiveness \\
MBS & CAACTG & MYB binding site involved in drought-inducibility \\
\hline
\end{tabular}

\subsection{PgSWEET17a Tobacco Transient Expression Analysis}

We compared the expression of PgSWEET17a in pomegranate fruits, leaves, buds and flowers (Figure 9B). PgSWEET17a expression was found to be significantly higher in leaves, buds and flowers $(p<0.05)$ than in fruits. To investigate the effect of PgSWEET17a on sugar concentration, the constructed pBI121-PgSWEET17a vector was infiltrated into tobacco (NB), and tobacco leaves were harvested on the third (3d), fourth (4d) and fifth (5d) days after dark incubation for PgSWEET17a expression and soluble sugar content determination (Figure 9A). Figure 9D shows that the expression of PgSEET17a was significantly higher on days four $(p<0.01)$ and five $(p<0.05)$ than in wild-type (WT) leaves, and there was no difference between leaves of WT and pBI121, and WT and 3d, indicating that PgSWEET17a was significantly induced in tobacco after infestation. Interestingly, fructose contents were significantly increased in leaves on the $3 \mathrm{~d}, 4 \mathrm{~d}$ and $5 \mathrm{~d}$ and sucrose and glucose contents were both remarkedly decreased (Figure 9C).

(A)
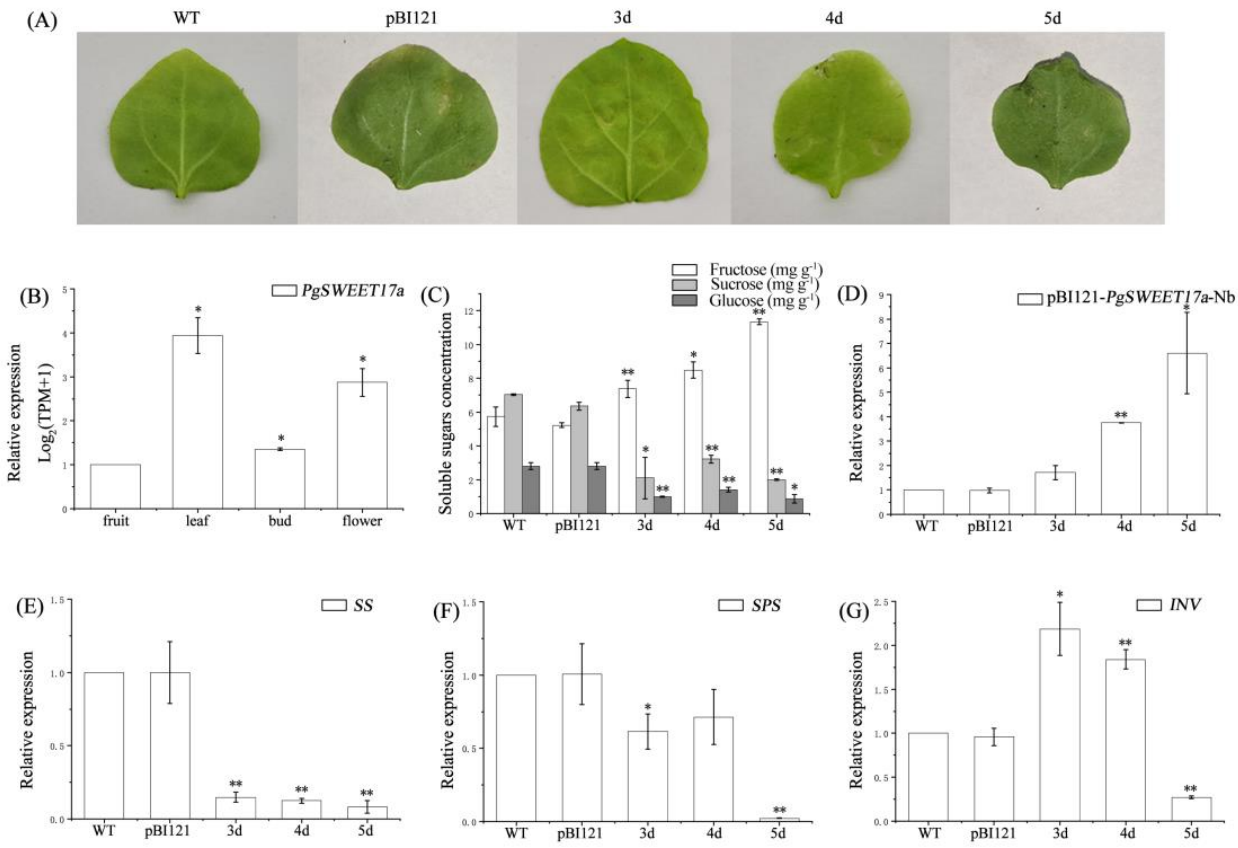

Figure 9. Tobacco transient expression results. Images of wild-type (WT), pBI121- overexpression and $P g S W E E T 17 a$-overexpression tobacco leaves (A). PgSWEET17a expression of different organs (B). Changes in soluble sugar concentration (C), PgSWEET17a expression (D), SS expression (E), SPS (F) and INV (G) in WT, pBI121-overexpression and PgSWEET17a-overexpression tobacco leaves. "** indicates significant differences at the 0.05 level, " $* * *$ indicate significant differences at the 0.01 level. 
In addition, we identified the expression of the sugar metabolism related enzymes $S S, S P S$ and INV genes in tobacco leaves. The SS transcript level, was significantly lower than that of WT on all three days $(p<0.01$, Figure 9E). SPS gene expression in leaves on the $3 \mathrm{~d}(p<0.05)$ and $5 \mathrm{~d}(p<0.01)$ were significantly lower than WT (Figure 9F). INV expression levels on the $3 \mathrm{~d}(p<0.05)$ and $4 \mathrm{~d}(p<0.01)$ were both significantly higher than WT, while on the $5 \mathrm{~d}$ it was significantly lower than WT (Figure 9G). There was no difference in expression of these genes between WT and pBI121(Figure 9). Overall, with the overexpression of PgSWEET17a, the expression of SS and SPS was induced to be downregulated in tobacco leaves, while the $I N V$ expression was significantly induced to be up-regulated at first and significantly decreased at a later stage.

\section{Discussion}

In higher plants, soluble sugars are mainly in the form of sucrose, glucose and fructose [47]. Sugar transport and distribution play an important role in promoting plant growth and development and crop yield improvement. The efficient regulation and distribution of sugar transport from source to sink organs through the phloem in plant tissues is one of the major determinants of plant growth [48]. Members of the SWEET family regulate the transport of different sugars through the cell membrane and control the sugars distribution inside and outside the cell. Currently, SWEETs genes are widely found in higher eukaryotes, as well as in protozoa, metazoans, fungi, bacteria and archaea [22]. The diversity of SWEETs in higher plants also reflects their different functions in growth, development and physiological processes, including pollen, seed and fruit development and nectar production $[9,13,23]$. The SWEETs family of genes has been found in many plants, such as 17, 21, 29, 17, 23, 13, 52, and 16 in Arabidopsis, rice, tomato, grape, sorghum, tea, soybean, and litchi, respectively $[8,12,13,49-53]$. Previous reports on the pomegranate SWEET family were missing. Now, three pomegranate genomes including 'Dabenzi', 'Tunisa' and 'Taishanhong' have been reported [40-42], which provides a reference for the identification and analysis of pomegranate SWEET genes and the functional exploration in this study.

Twenty candidate PgSWEET family members were identified and analyzed. The analysis showed that there were tandem duplications of PgSWEET genes. It has been suggested that the expansion of SWEET genes in dicots is mainly the result of recent duplication events, especially tandem duplication [54]. Based on the phylogenetic tree reconstructed using pomegranate, Arabidopsis and grape SWEETs, the analysis showed that the PgSWEETs family was divided into four subfamilies, namely Clade I, II, III and IV. Different PgSWEET genes have different structures, with little variation in the number of exons and large variation in the length of introns, and a tendency to grow as the evolutionary tree branches from the outside to the inside. The last common ancestor of angiosperm SWEETs was speculated to have 6 exons and gene size variation was presumed to be caused by intron insertion [54]. Most SWEET genes in pomegranate, Arabidopsis and grape have a 1:1:1 relationship in number, which may be due to a $\gamma$ WGD (whole genome duplication) event in angiosperms [40].

A specific expansion subclade consisting of five PgSWEETs was present in Clade I, and traces of PgSWEET1 $a$ and $1 b$ expression were found based on transcriptomic results, which may be due to pseudogenization as a result of duplication events [55]. PgSWEET1c was highly expressed in roots, as well as expressed in both bisexual and functional male flower buds. PgSWEET1d was only higher expressed in roots. It was speculated that PgSWEET1c and $1 d$ were important in glucose transport from roots [8]. PgSWEET1e was induced with the development of functional male flowers, implying that it provides nutrition to the developing gametophyte or nectaries [8]. AtSWEET15 in Arabidopsis has been validated to be associated with plant senescence and to respond to adversity stress to maintain normal plant growth [21,56]. The induction of some genes involved in plant defense mechanisms is an integral part of the leaf senescence program [57]. PgSWEET16s and 17s have high sequence similarity and belong to the Clade IV, a branch mainly involved in fructose 
transport. PgSWEET16b was highly expressed in leaves and PgSWEET17a was mainly expressed specifically in leaves, skins and roots, suggesting that they regulate fructose content in these tissues $[14,20,30]$.

Indeed, foliar application of tripotassium phosphate at the time of pomegranate fruit color turning stage increased the fruit soluble sugar content. To preliminarily investigate the mechanism of regulation of sugar metabolism by tripotassium phosphate in pomegranate, we determined the expression of the PgSWEETs family in pomegranate leaves and fruits. On September 20, all genes were highly expressed except for PgSWEET1e, 2, 10 and 16a. Later, in the control group, most PgSWEETs had low transcript levels, while PgSWEET1e, 2, 10 and $16 a$ were expressed at high levels, and PgSWEET9, $16 b$ and $17 b$ were expressed at slightly higher levels on 10 October, suggesting that these genes may play an important role in determining fruit quality and yield at fruit ripening [53]. Foliar spraying of tripotassium phosphate significantly increased PgSWEET2, 5, 9, 12, 16b and $17 \mathrm{~b}$ compared to control on 30 September, and increased PgSWEET1e and 16 expression on 10 October in fruit. These genes produced high expressed levels in response to tripotassium phosphate in fruit. In pomegranate leaf, PgSWEET1e, 2, 9, 11,16a and 17a expression in treatment group was significantly lower than control on September 30, and most genes expression was significantly higher than control except for PgSWEET3 and 15 on 10 October. These implied the spatiotemporal expression specificity of PgSWEETs. PgSWEETs had high expression levels in response to tripotassium phosphate in leaf. PgSWEET15 in leaf after tripotassium phosphate treatment was significantly decreased, which might be due to the delayed leaf senescence [21]. These suggest that SWEETs are involved in regulating the redistribution of soluble sugars within pomegranate tissues in response to tripotassium phosphate to influence plant growth.

Sugar regulates juvenile and floral signaling through energy sources, osmoregulation, and signaling molecules, and sugar signaling has been proven to be important for coordinating developmental transitions in interaction with phytohormones [38]. ABA is a floral induction inhibitor whose components of the synthesis and transduction pathway are allelic to mutations that affect sugar signaling [58,59]. Meanwhile, glucose can enhance ABA concentration and ABA-biosynthesis-related gene expression [60]. Ethylene has regulatory effects on juvenile development and flower induction [61]. In addition, ethylene can affect the sensitivity of plants to sugars, and the application of ethylene precursors can reduce the sensitivity of plants to glucose [62]. It was clear that the soluble sugar content of flower buds was significantly induced after hormone treatment. We determined the expression of several important PgSWEETs and found that hormone treatment has an effect on the sugar transporter protein. Six PgSWEETs were differentially expressed in bisexual and functional male flowers and showed different trends in response to different hormones, suggesting that these genes may be involved in stress response or in the response process of different phytohormone signal transduction in pomegranate development.

It was shown that most SWEET proteins are localized on the plasma membrane and may be the main sites for regulating sugar flux $[63,64]$. SWEET proteins have been identified as bidirectional transporter proteins that regulate cellular uptake and efflux of sugars, but there are differences in substrate specificity among SWEETs [7,65]. In Arabidopsis, AtSWEET17 is located in the vacuole membrane and can regulate changes in fructose levels in leaves. Under low nitrogen conditions, large amounts of sugars accumulate in plant tissues and large amounts of monosaccharides (glucose and fructose) accumulate in the vesicles via the vacuole monosaccharide transporter TMT, from where AtSWEET17 apparently exports a portion of fructose to the cytoplasm; this function is impaired in the mutant sweet17, leading to a specific accumulation of fructose in the tissues [14]. In higher plants, the rapid accumulation of soluble sugars can cause metabolic changes. In wild-type tobacco, sucrose is mainly distributed in the cytoplasm, with up to $98 \%$ hexose in the vacuole, and in transgenic tobacco, the hexose content is higher than in wild-type plants, again with 97-98\% hexose stored in the vacuole; in short, the vacuole in the leaves actively take up the transporters of glucose and fructose at high concentration gradients [66]. 
In this study, overexpression of PgSWEET17a caused an increase in fructose content in transgenic tobacco, while both sucrose and glucose content decreased. It is speculated that $P g S W E E T 17 a$ is involved in the transport of fructose, sucrose and glucose. In addition, PgSWEET17a expression level was affected by exogenous hormones in pomegranate flower buds. We found that the expression of PgSWEET17a was significantly higher in bisexual flower buds than in functional male flower buds during the critical period of sterile flower abortion, i.e., the P2 stage. It is hypothesized that PgSWEET17a plays a role in the formation of fertile flowers in buds.

Sugar metabolism is influenced by a variety of enzymes and their related genes. During fruit development, sucrose transport from source tissues to sink organs through the phloem requires SS, INV and SPS to complete the conversion between sucrose, fructose and glucose, and to create a sucrose concentration gradient between source and sink to drive sugar transport. SS plays an important role in plant cellulose synthesis, starch synthesis and sucrose transport, and its role in overexpressing OsSUS3 and AtSUS in plants and citrus fruits has been verified [67-69]. Furthermore, sucrose inversion catalyzed by INV requires two molecules of ATP, while only one molecule of PPi is consumed via SS, which can be recycled, making the SS pathway more energy-efficient [70]. Related evidence suggests that overexpression of INV in potato plants consumes too much ATP, and although the up-regulated INV promotes sucrose inversion, it also limits starch accumulation in the stem mass [71].

\section{Material and Methods}

\subsection{Identification and Sequence Analysis of PgSWEETs}

HMM model files were constructed based on the SWEET gene family sequence alignment file (PF03083) downloaded from the Pfam database, and protein sequences containing the conserved structural domain of SWEET (E-value $\left.\leq \mathrm{e}^{-10}\right)$ in the pomegranate genome file [40] were screened using SelectHMM (https:/ / github.com/Redpome/SelectHMM, accessed on 1 February 2022). Then, prediction of physicochemical properties of PgSWEET protein sequences using the online software Protparam (https:/ / web.expasy.org/protparam/, accessed on 1 February 2022).

\subsection{Phylogenetic Tree Construction}

The protein sequences of the screened PgSWEETs were aligned with Arabidopsis and grape SWEETs using MAFFT7.487 [72], and the protein sequence alignment was transformed into the corresponding codon alignment using pal2nal script [73] to construct a phylogenetic tree using iqtree [74]. The evolutionary tree is visualized using figtree software (http: / / tree.bio.ed.ac.uk/software/figtree/, accessed on 1 February 2022).

\subsection{PgSWEETs Gene Structure Analysis}

Gene structure information of the PgSWEET gene family, including introns, exons and upstream and downstream sequences, was obtained from the pomegranate genome annotation file, and the results were presented using the online software GSDS (http: / / gsds.cbi.pku.edu.cn, accessed on 1 February 2022).

\subsection{PgSWEET Gene Family RNA-Seq Analysis}

To analyze expression patterns of PgSWEETs in different pomegranate tissues and organs, the published transcriptome data (Table 3) were downloaded from NCBI (http: / / www.ncbi.nlm.nih.gov/, accessed on 1 February 2022). The transcriptomic data were calculated and analyzed using Kallisto v0.44.0 software (CA, USA), and the resulting values were transformed into $\log _{2}(\mathrm{TPM}+1)$ and finally clustered for heat mapping using pheatmap in $\mathrm{R}$ [75]. 


\subsection{Plant Material and Tripotassium Phosphate Foliar Fertilization}

The pomegranate fruits and leaves were collected from September to October 2020 in Hu Gang Pomegranate Garden, Liuhe District, Nanjing, Jiangsu Province. The treatments for the field trial were as follows: foliar sprays were applied to uniformly growing pomegranate trees, with prue water as the control group (CK) and $500 \mathrm{ppm}$ concentration of tripotassium phosphate $\left(\mathrm{K}_{3} \mathrm{PO}_{4}\right)$ as the treatment group. Five pomegranate trees were selected for each treatment. Spraying began on 20 September 2020 (pomegranate fruit color turning stage), and was applied at 10-day intervals for a total of three sprays. The level of spraying was until the leaves dripped on both sides. The pomegranates were picked before each spraying, 4 times in total. Pomegranate fruit and leaves of consistent growth and free of pests and diseases were picked from each tree in all directions. The last picking was done on 20 October (fruit ripening period). The fruit seeds were mixed well after each picking, treated with liquid nitrogen and stored in $-80^{\circ} \mathrm{C}$ refrigerator for backup. (No permission was required for sample collection.)

\subsection{Plant Material and Exogenous Hormone Treatments}

"Taishanhong" pomegranate was selected as the experimental cultivar. Three plant growth regulators (CK: pure water, $100 \mathrm{mg} / \mathrm{L}$ 6-BA, $20 \mathrm{mg} / \mathrm{L} \mathrm{IBA}$ and $1000 \mathrm{mg} / \mathrm{L} \mathrm{PP}_{333}$ ) were selected and sprayed on the leaves as follows: three sprays were applied on 5 October 2019 (dormant period), 15 April 2020 (spring leaf expansion period) and 10 May (initial flowering to full flowering period). Flower buds of bisexual and functional male flowers of pomegranate at different stages of development were collected. Flower buds were divided into three stages according to their longitudinal diameter: 3-5 mm (P1), 5.1-13 mm (P2) and $13.1-25 \mathrm{~mm}$ (P3). (No permission was required for sample collection.)

\subsection{Total Soluble Sugar Content Determination}

Total soluble sugar of fruits, leaves and buds was determined using the anthronesulfuric acid colorimetry method [76].

\subsection{RNA Isolation and Gene Clone}

The reagents for the test included laboratory-preserved overexpression vector $\mathrm{pBI} 121$, $\mathrm{DH} \alpha$ (Beijing Prime Tech Biotechnology Co., Ltd., Beijing, China), GV3101 (Shanghai Viearth Biotechnology Co., Ltd., Shanghai, China), One-Step Cloning Kit and $2 \times$ Taq Plus Master Mix enzyme (Nanjing Novizan Biotechnology Co., Ltd., Nanjing, China), Polysaccharide Polyphenol Plant RNA Extraction Kit (Beijing Tiangen Biochemical Technology Co., Ltd., Beijing, China), Reverse Transcription Kit (Takara Company, Tokyo, Japan), and PrimeScriptTM RT reagent Kit with gDNA Eraser (Takara Company), DNA Maker and Glue cutting Recovery Kit (Beijing Qingke Biotechnology Co., Ltd., Beijing, China). The primers used in the experiments were synthesized by Shanghai Bioengineering Compony (Table 5).

The PCR amplification system was: $25 \mu \mathrm{L} 2 \times$ Taq Plus Master Mix; $1 \mu \mathrm{L}$ forwardprimer; $1 \mu \mathrm{L}$ reverseprimer; $2 \mu \mathrm{L}$ DNA template; $21 \mu \mathrm{L}$ nuclease-free ddH2O. PCR program settings were: $95{ }^{\circ} \mathrm{C}$ : $3 \mathrm{~min} ; 95^{\circ} \mathrm{C}: 15 \mathrm{~s} ; 52{ }^{\circ} \mathrm{C}: 45 \mathrm{~s} ; 72{ }^{\circ} \mathrm{C}$ : $1 \mathrm{~min}$; for a total of 35 cycles; $72{ }^{\circ} \mathrm{C}$ : $5 \mathrm{~min} ; 4^{\circ} \mathrm{C}$ : save. The PCR products were separated by agar gel electrophoresis, the target fragments were recovered by gel cutting, and then the CDS sequences of PgSWEET17a gene were obtained by amplification, ligation and transformation steps, and translated into amino acid sequences using Translate online software (https:/ /web.expasy.org/translate/, accessed on 9 December 2021). 
Table 5. Primers for the gene cloning, transient expression and qRT-PCR.

\begin{tabular}{|c|c|c|}
\hline Primer & Primer Sequence $\left(5^{\prime}-3^{\prime}\right)$ & Annotation \\
\hline PgSWEET17a & $\begin{array}{c}\text { F: ATGAGAAGCTTGAGT } \\
\text { R: TTAATTATCATTTTGTTCCCT }\end{array}$ & Gene clone \\
\hline GFP-PgSWEET17a & $\begin{array}{c}\text { F: gagaacacgggggactctagaATGAGAAGCTTGAGT } \\
\text { R: gccettgctcaccatggatccTTAATTATCATTTTGTTCCCT }\end{array}$ & Transient expression \\
\hline qRT-PgSWEET17a & $\begin{array}{c}\text { F: GCCGTTCTTCCTCTCGTT } \\
\text { R: TTTTGTTCCCTGCGATGGCT }\end{array}$ & Gene expression \\
\hline$q R T-S S$ & $\begin{array}{l}\text { F:ATCAAGTTCCGGCCTTGGAG } \\
\text { R:CCTCAGTGAATGTCTCCATG }\end{array}$ & Gene expression \\
\hline$q R T-S P S$ & $\begin{array}{l}\text { F:GGAATTACAGCCCATACGAG } \\
\text { R:AAGTTCTGGGTGAGCAAA }\end{array}$ & Gene expression \\
\hline$q R T-I N V$ & $\begin{array}{l}\text { F:CTCCACGACCCATTACAC } \\
\text { R:GGAAACTCCCTGAGATACA }\end{array}$ & Gene expression \\
\hline PgActin & $\begin{array}{l}\text { F: AGTCCTCTTCCAGCCATCTC } \\
\text { R: CACTGAGCACAATGTTTCCA }\end{array}$ & Gene expression \\
\hline NtActin & $\begin{array}{l}\text { F:CAAGGAAATCACCGCTTTGG } \\
\text { R:AAGGGATGCGAGGATGGA }\end{array}$ & Gene expression \\
\hline
\end{tabular}

\subsection{PgSWEET17a Bioinformatics Analysis}

The basic physicochemical properties of the PgSWEET17a protein sequence were analyzed using the online tool ProtParam (https: / / web.expasy.org/protparam/, accessed on 9 December 2021). Its subcellular localization was predicted using the online tool Plant-mPLoc (http: / / www.csbio.sjtu.edu.cn/bioinf/plant-multi/, 9 December 2021).

Homologous protein search was performed using Blastp (http:/ / www.ncbi.nlm.nih. gov /BLAST / , accessed on 9 December 2021). Multiple sequence alignment and visualization of amino acid sequences were performed using DNAMAN and WebLogo, respectively.

\subsection{PgSWEET7a Subcellular Location Analysis}

The correct sequence PgSWEET17a obtained by cloning was ligated to the pBI121 vector and the carbon end was fused to green fluorescent protein (GFP). The recombinant plasmid was then introduced into Agrobacterium tumefaciens GV3101 by the freeze-thaw method. The pBI121-GFP was used as a control, and both the recombinant plasmid and the control plasmid were infiltrated with young leaves of $N$. benthamiana by the Agrobacteriummediated method. After dark and light cultivation for $24 \mathrm{~h}$ respectively, fluorescence images were collected under a confocal microscope.

\subsection{PgSWEET17a Cis-Acting Elements Analysis}

A $1500 \mathrm{bp}$ promoter sequence upstream of the initial codon was extracted from the PgSWEET17a genomic sequence and used to predict cis-regulatory elements using PlantCARE (http:/ / bioinformatics.psb.ugent.be/webtools/plantcare/html/, accessed on 9 December 2021).

\subsection{Real-Time Fluorescence Quantitative Analysis (qRT-PCR)}

qRT-PCR was used to determine the expression pattern of PgSWEETs in pomegranate fruits, leaves, flower buds and tobacco leaves. The fluorescent dye used was the BioEasy Master Mix Plus (SYBR Green, Beijing Bioteke Biotechnology Co., Ltd., Beijing, China), and the reaction procedure was: $95^{\circ} \mathrm{C}: 3 \mathrm{~min} ; 95^{\circ} \mathrm{C}: 30 \mathrm{~s} ; 60^{\circ} \mathrm{C}: 15 \mathrm{~s} ; 72{ }^{\circ} \mathrm{C}: 20 \mathrm{~s}, 40$ cycles from step 2 to step 4. The pomegranate PgActin and NtActin genes were used as an internal reference and three biological replicates were performed for each treatment. The data were quantified using the $2^{-\Delta \Delta \mathrm{Ct}}$ method. Primers used for qRT-PCR are shown in Tables 2 and S1. 


\subsection{Agrobacterium Infiltration}

The PgSWEET17a recombinant plasmid with GFP tag was transferred into Agrobacterium tumefaciens GV3101, activated and inoculated in $50 \mathrm{~mL}$ LB liquid medium, incubated in a shaker at $28^{\circ} \mathrm{C}, 220 \mathrm{r} / \mathrm{min}$ for $12 \mathrm{~h}$. After removal, the bacterium was collected by centrifugation at $4{ }^{\circ} \mathrm{C}, 4000 \mathrm{rpm} / \mathrm{min}$ for $10 \mathrm{~min}$, and a resuspension solution was prepared $\left(10 \mathrm{mmol} / \mathrm{L} \mathrm{MES}+10 \mathrm{mmol} / \mathrm{L} \mathrm{MgCl} 2 \cdot 6 \mathrm{H}_{2} \mathrm{O}+100 \mathrm{mmol} / \mathrm{L} \mathrm{AS}, \mathrm{pH}\right.$ 5.6) 10 The bacteria were washed and resuspended at room temperature for $2 \mathrm{~h}$. The bacteria were injected into tobacco leaves, sampled after infestation, and stored at $-80^{\circ} \mathrm{C}$. (Tobacco seeds were kept in our laboratory).

\section{Conclusions}

In this study, we identified 20 pomegranate SWEET genes. Phylogenetic tree analysis revealed that PgSWEETs were divided into four subgroups and that genome-wide replication events and tandem replication together contributed to the evolution of the SWEET gene family. Based on the available transcriptomic data, we identified certain SWEETs that play important roles in pomegranate growth and development. Foliar application of tripotassium phosphate increased the sugar content of pomegranate leaves and fruits, and hormone treatment also affected sugar metabolism in pomegranate bisexual and functional male flowers. qRT-PCR analysis showed that PgSWEETs responded to both tripotassium phosphate and hormone treatments to varying degrees. Subcellular localization revealed that PgSWEET17a protein was localized on the cell membrane. The transient expression technique revealed that PgSWEET17a is involved in the transport of fructose, sucrose and glucose, with positive regulation of fructose and negative regulation of sucrose and glucose.

Supplementary Materials: The following supporting information can be downloaded at: https: / / www.mdpi.com/article/10.3390/ijms23052471/s1.

Author Contributions: Conceptualization, X.Z. (Xinhui Zhang), S.W. and Z.Y.; test sample processing and collection, X.Z. (Xinhui Zhang), Y.R. and B.L.; experimental operation, X.Z. (Xinhui Zhang), S.W., C.G. and Y.F.; writing — original draft preparation, X.Z. (Xinhui Zhang); writing-review and editing, X.Z. (Xinhui Zhang), S.W. and X.Z. (Xueqing Zhao); visualization, X.Z. (Xinhui Zhang); project administration, Z.Y. All authors have read and agreed to the published version of the manuscript.

Funding: This work was supported by the Initiative Project for Talents of Nanjing Forestry University (GXL2014070, GXL2018032), the Doctorate Fellowship Foundation of Nanjing Forestry University, and the Priority Academic Program Development of Jiangsu High Education Institutions (PAPD), the National Natural Science Foundation of China (31901341), and the Natural Science Foundation of Jiangsu Province (BK20180768). These funding bodies took part in the design of the study and collection, analysis, and interpretation of data, and the writing of the manuscript, as well as in the open access payment.

Institutional Review Board Statement: Not applicable.

Informed Consent Statement: Informed consent was obtained from all subjects involved in the study.

Data Availability Statement: The data presented in this study are available on request from the corresponding author and the public pomegranate transcriptomes presented in this study are available in insert article.

Conflicts of Interest: The authors declare no conflict of interest.

\section{References}

1. Falchi, R.; Bonghi, C.; Drincovich, M.F.; Famiani, F.; Lara, M.V.; Walker, R.P.; Vizzotto, G. Sugar Metabolism in Stone Fruit: Source-Sink Relationships and Environmental and Agronomical Effects. Front. Plant Sci. 2020, 11, 1820. [CrossRef] [PubMed]

2. Zhang, C.; Bian, Y.; Hou, S.; Li, X. Sugar transport played a more important role than sugar biosynthesis in fruit sugar accumulation during Chinese jujube domestication. Planta 2018, 248, 1187-1199. [CrossRef] [PubMed]

3. Julius, B.T.; Leach, K.A.; Tran, T.M.; Mertz, R.A.; Braun, D.M. Sugar Transporters in Plants: New Insights and Discoveries. Plant Cell Physiol. 2017, 58, 1442-1460. [CrossRef] 
4. Ruan, Y.-L. Sucrose Metabolism: Gateway to Diverse Carbon Use and Sugar Signaling. Annu. Rev. Plant Biol. 2014, 65, 33-67. [CrossRef] [PubMed]

5. Chen, L.-Q. SWEET sugar transporters for phloem transport and pathogen nutrition. New Phytol. 2014, 201, 1150-1155. [CrossRef]

6. Chandran, D. Co-option of developmentally regulated plant SWEET transporters for pathogen nutrition and abiotic stress tolerance. IUBMB Life 2015, 67, 461-471. [CrossRef]

7. Chen, L.Q.; Qu, X.Q.; Hou, B.H.; Sosso, D.; Osorio, S.; Fernie, A.R.; Frommer, W.B. Sucrose efflux mediated by SWEET proteins as a key step for phloem transport. Science 2012, 335, 207-211. [CrossRef]

8. Chen, L.Q.; Hou, B.H.; Lalonde, S.; Takanaga, H.; Hartung, M.L.; Qu, X.Q.; Guo, W.J.; Kim, J.G.; Underwood, W.; Chaudhuri, B.; et al. Sugar transporters for intercellular exchange and nutrition of pathogens. Nature 2010, 468, 527-532. [CrossRef]

9. Ayre, B.G. Membrane-transport systems for sucrose in relation to whole-plant carbon partitioning. Mol. Plant 2011, 4, 377-394. [CrossRef]

10. Slewinski, T.L. Diverse Functional Roles of Monosaccharide Transporters and their Homologs in Vascular Plants: A Physiological Perspective. Mol. Plant 2011, 4, 641-662. [CrossRef]

11. Hamada, M.; Wada, S.; Kobayashi, K.; Satoh, N. Ci-Rga, a gene encoding an MtN3/saliva family transmembrane protein, is essential for tissue differentiation during embryogenesis of the ascidian Ciona intestinalis. Differentiation 2005, 73, 364-376. [CrossRef] [PubMed]

12. Yuan, M.; Zhao, J.; Huang, R.; Li, X.; Xiao, J.; Wang, S. Rice MtN3/saliva/SWEET gene family: Evolution, expression profiling, and sugar transport. J. Integr. Plant Biol. 2014, 56, 559-570. [CrossRef] [PubMed]

13. Chong, J.; Piron, M.C.; Meyer, S.; Merdinoglu, D.; Bertsch, C.; Mestre, P. The SWEET family of sugar transporters in grapevine: VvSWEET4 is involved in the interaction with Botrytis cinerea. J. Exp. Bot. 2014, 65, 6589-6601. [CrossRef]

14. Chardon, F.; Bedu, M.; Calenge, F.; Klemens, P.A.W.; Spinner, L.; Clement, G.; Chietera, G.; Léran, S.; Ferrand, M.; Lacombe, B.; et al. Leaf Fructose Content Is Controlled by the Vacuolar Transporter SWEET17 in Arabidopsis. Curr. Biol. 2013, 23, 697-702. [CrossRef] [PubMed]

15. Lin, I.W.; Sosso, D.; Chen, L.Q.; Gase, K.; Kim, S.G.; Kessler, D.; Klinkenberg, P.M.; Gorder, M.K.; Hou, B.H.; Qu, X.Q. Nectar secretion requires sucrose phosphate synthases and the sugar transporter SWEET9. Nature 2014, 508, 546-549. [CrossRef] [PubMed]

16. Antony, G.; Zhou, J.; Huang, S.; Li, T.; Liu, B.; White, F.; Yang, B. Rice xa13 Recessive Resistance to Bacterial Blight Is Defeated by Induction of the Disease Susceptibility Gene Os-11N3. Plant Cell 2010, 22, 3864-3876. [CrossRef] [PubMed]

17. Wang, J.; Yan, C.; Li, Y.; Hirata, K.; Yamamoto, M.; Yan, N.; Hu, Q. Crystal structure of a bacterial homologue of SWEET transporters. Cell Res. 2014, 24, 1486-1489. [CrossRef] [PubMed]

18. Moran Lauter, A.N.; Peiffer, G.A.; Yin, T.; Whitham, S.A.; Cook, D.; Shoemaker, R.C.; Graham, M.A. Identification of candidate genes involved in early iron deficiency chlorosis signaling in soybean (Glycine max) roots and leaves. BMC Genom. 2014, 15, 702. [CrossRef]

19. Zhang, J.; Huguet-Tapia, J.C.; Hu, Y.; Jones, J.; Wang, N.; Liu, S.; White, F.F. Homologues of CsLOB1 in citrus function as disease susceptibility genes in citrus canker. Mol. Plant Pathol. 2017, 18, 798-810. [CrossRef]

20. Guo, W.J.; Nagy, R.; Chen, H.Y.; Pfrunder, S.; Yu, Y.C.; Santelia, D.; Frommer, W.B.; Martinoia, E. SWEET17, a facilitative transporter, mediates fructose transport across the tonoplast of Arabidopsis roots and leaves. Plant Physiol. 2014, 164, 777-789. [CrossRef]

21. Seo, P.J.; Park, J.-M.; Kang, S.; Kim, S.-G.; Park, C.-M. An Arabidopsis senescence-associated protein SAG29 regulates cell viability under high salinity. Planta 2010, 233, 189-200. [CrossRef] [PubMed]

22. Yuan, M.; Wang, S. Rice MtN3/saliva/SWEET family genes and their homologs in cellular organisms. Mol. Plant 2013, 6, 665-674. [CrossRef] [PubMed]

23. Sosso, D.; Luo, D.; Li, Q.B.; Sasse, J.; Yang, J.; Gendrot, G.; Suzuki, M.; Koch, K.E.; McCarty, D.R.; Chourey, P.S.; et al. Seed filling in domesticated maize and rice depends on SWEET-mediated hexose transport. Nat. Genet. 2015, 47, 1489-1493. [CrossRef] [PubMed]

24. Engel, M.L.; Holmes-Davis, R.; McCormick, S. Green sperm. Identification of male gamete promoters in Arabidopsis. Plant Physiol. 2005, 138, 2124-2133. [CrossRef]

25. Salts, Y.; Sobolev, I.; Chmelnitsky, I.; Shabtai, S.; Barg, R. Genomic structure and expression of Lestd1, a seven-transmembranedomain proteon-encoding gene specically expressed in tomato pollen. Isr. J. Plant Sci. 2005, 53, 79-88. [CrossRef]

26. Bock, K.W.; Honys, D.; Ward, J.M.; Padmanaban, S.; Nawrocki, E.P.; Hirschi, K.D.; Twell, D.; Sze, H. Integrating membrane transport with male gametophyte development and function through transcriptomics. Plant Physiol. 2006, 140, 1151-1168. [CrossRef]

27. Guan, Y.-F.; Huang, X.-Y.; Zhu, J.; Gao, J.-F.; Zhang, H.-X.; Yang, Z.-N. Ruptured Pollen GRAIN1, a member of the MtN3/saliva gene family, is crucial for exine pattern formation and cell integrity of microspores in Arabidopsis. Plant Physiol. 2008, 147, 852-863. [CrossRef]

28. Sun, M.-X.; Huang, X.-Y.; Yang, J.; Guan, Y.-F.; Yang, Z.-N. Arabidopsis RPG1 is important for primexine deposition and functions redundantly with RPG2 for plant fertility at the late reproductive stage. Plant Reprod. 2013, 26, 83-91. [CrossRef] 
29. Römer, P.; Recht, S.; Straus, T.; Elsaesser, J.; Schornack, S.; Boch, J.; Wang, S.; Lahaye, T. Promoter elements of rice susceptibility genes are bound and activated by specific TAL effectors from the bacterial blight pathogen, Xanthomonas oryzae pv. oryzae. New Phytol. 2010, 187, 1048-1057. [CrossRef]

30. Klemens, P.A.; Patzke, K.; Deitmer, J.; Spinner, L.; Le Hir, R.; Bellini, C.; Bedu, M.; Chardon, F.; Krapp, A.; Neuhaus, H.E. Overexpression of the vacuolar sugar carrier AtSWEET16 modifies germination, growth, and stress tolerance in Arabidopsis. Plant Physiol. 2013, 163, 1338-1352. [CrossRef]

31. Paller, C.J.; Pantuck, A.; Carducci, M.A. A review of pomegranate in prostate cancer. Prostate Cancer Prostatic Dis. 2017, 20, 265-270. [CrossRef] [PubMed]

32. Sharma, P.; McClees, S.F.; Afaq, F. Pomegranate for prevention and treatment of cancer: An update. Molecules 2017, $22,177$. [CrossRef]

33. Zarfeshany, A.; Asgary, S.; Javanmard, S.H. Potent health effects of pomegranate. Adv. Biomed. Res. 2014, 3, 100. [CrossRef] [PubMed]

34. Wang, D.; Özen, C.; Abu-Reidah, I.M.; Chigurupati, S.; Patra, J.K.; Horbanczuk, J.O.; Jóźwik, A.; Tzvetkov, N.T.; Uhrin, P.; Atanasov, A.G. Vasculoprotective Effects of Pomegranate (Punica granatum L.). Front. Pharmacol. 2018, 9, 544. [CrossRef] [PubMed]

35. Melgarejo, P.; Salazar, D.M.; Artes, F. Organic acids and sugars composition of harvested pomegranate fruits. Eur. Food Res. Technol. 2000, 211, 185-190. [CrossRef]

36. Meletis, K.; Tsaniklidis, G.; Papadakis, I.E.; Vemmos, S.N. Sucrose synthesis in Unpollinated ovaries of pomegranate (Punica granatum L.), as well as, in reproductive and vegetative shoot apices. Biologia 2019, 74, 111-117. [CrossRef]

37. Xing, L.B.; Zhang, D.; Li, Y.M.; Shen, Y.W.; Zhao, C.P.; Ma, J.J.; An, N.; Han, M.Y. Transcription Profiles Reveal Sugar and Hormone Signaling Pathways Mediating Flower Induction in Apple (Malus domestica Borkh.). Plant Cell Physiol. 2015, 56, $2052-2068$. [CrossRef]

38. Matsoukas, I.G. Interplay between sugar and hormone signaling pathways modulate floral signal transduction. Front. Genet. 2014, 5, 2018. [CrossRef]

39. Hernández, J.A.; Díaz-Vivancos, P.; Acosta-Motos, J.R.; Alburquerque, N.; Martínez, D.; Carrera, E.; García-Bruntón, J.; BarbaEspín, G. Interplay among Antioxidant System, Hormone Profile and Carbohydrate Metabolism during Bud Dormancy Breaking in a High-Chill Peach Variety. Antioxidants 2021, 10, 560. [CrossRef]

40. Yuan, Z.; Fang, Y.; Zhang, T.; Fei, Z.; Han, F.; Liu, C.; Liu, M.; Xiao, W.; Zhang, W.; Wu, S.; et al. The pomegranate (Punica granatum L.) genome provides insights into fruit quality and ovule developmental biology. Plant Biotechnol. J. 2018, 16, 1363-1374. [CrossRef] [PubMed]

41. Luo, X.; Li, H.; Wu, Z.; Yao, W.; Zhao, P.; Cao, D.; Yu, H.; Li, K.; Poudel, K.; Zhao, D. The pomegranate (Punica granatum L.) draft genome dissects genetic divergence between soft-and hard-seeded cultivars. Plant Biotechnol. J. 2019, 18, 955-968. [CrossRef] [PubMed]

42. Qin, G.; Xu, C.; Ming, R.; Tang, H.; Guyot, R.; Kramer, E.M.; Hu, Y.; Yi, X.; Qi, Y.; Xu, X. The pomegranate (Punica granatum L.) genome and the genomics of punicalagin biosynthesis. Plant J. 2017, 91, 1108-1128. [CrossRef] [PubMed]

43. Ophir, R.; Sherman, A.; Rubinstein, M.; Eshed, R.; Sharabi Schwager, M.; Harel-Beja, R.; Bar-Ya'akov, I.; Holland, D. Singlenucleotide polymorphism markers from de-novo assembly of the pomegranate transcriptome reveal germplasm genetic diversity. PLoS ONE 2014, 9, e88998. [CrossRef]

44. Chen, L.; Zhang, J.; Li, H.; Niu, J.; Xue, H.; Liu, B.; Wang, Q.; Luo, X.; Zhang, F.; Zhao, D.; et al. Transcriptomic Analysis Reveals Candidate Genes for Female Sterility in Pomegranate Flowers. Front. Plant. Sci. 2017, 8, 1430. [CrossRef]

45. Xuan, Y.H.; Hu, Y.B.; Chen, L.Q.; Sosso, D.; Ducat, D.C.; Hou, B.H.; Frommer, W.B. Functional role of oligomerization for bacterial and plant SWEET sugar transporter family. Proc. Natl. Acad. Sci. USA 2013, 110, E3685-E3694. [CrossRef] [PubMed]

46. Tao, Y.; Cheung, L.S.; Li, S.; Eom, J.-S.; Chen, L.-Q.; Xu, Y.; Perry, K.; Frommer, W.B.; Feng, L. Structure of a eukaryotic SWEET transporter in a homotrimeric complex. Nature 2015, 527, 259-263. [CrossRef] [PubMed]

47. Ap Rees, T. Plant physiology. Virtue on both sides. Curr. Biol. 1994, 4, 557-559. [CrossRef]

48. Lemoine, R.; La Camera, S.; Atanassova, R.; Dédaldéchamp, F.; Allario, T.; Pourtau, N.; Bonnemain, J.-L.; Laloi, M.; CoutosThévenot, P.; Maurousset, L.; et al. Source-to-sink transport of sugar and regulation by environmental factors. Front. Plant Sci. 2013, 4, 272. [CrossRef]

49. Mizuno, H.; Kasuga, S.; Kawahigashi, H. The sorghum SWEET gene family: Stem sucrose accumulation as revealed through transcriptome profiling. Biotechnol. Biofuels 2016, 9, 127. [CrossRef]

50. Wang, L.; Yao, L.; Hao, X.; Li, N.; Qian, W.; Yue, C.; Ding, C.; Zeng, J.; Yang, Y.; Wang, X. Tea plant SWEET transporters: Expression profiling, sugar transport, and the involvement of CsSWEET16 in modifying cold tolerance in Arabidopsis. Plant Mol. Biol. 2018, 96, 577-592. [CrossRef]

51. Patil, G.; Valliyodan, B.; Deshmukh, R.; Prince, S.; Nicander, B.; Zhao, M.; Sonah, H.; Song, L.; Lin, L.; Chaudhary, J.; et al. Soybean (Glycine max) SWEET gene family: Insights through comparative genomics, transcriptome profiling and whole genome re-sequence analysis. BMC Genom. 2015, 16, 520. [CrossRef] [PubMed]

52. Xie, H.; Wang, D.; Qin, Y.; Ma, A.; Fu, J.; Qin, Y.; Hu, G.; Zhao, J. Genome-wide identification and expression analysis of SWEET gene family in Litchi chinensis reveal the involvement of LcSWEET2a/3b in early seed development. BMC Plant Biol. 2019, 19, 499. [CrossRef] [PubMed] 
53. Feng, C.-Y.; Han, J.-X.; Han, X.-X.; Jiang, J. Genome-wide identification, phylogeny, and expression analysis of the SWEET gene family in tomato. Gene 2015, 573, 261-272. [CrossRef] [PubMed]

54. Hu, W.; Hua, X.; Zhang, Q.; Wang, J.; Shen, Q.; Zhang, X.; Wang, K.; Yu, Q.; Lin, Y.R.; Ming, R.; et al. New insights into the evolution and functional divergence of the SWEET family in Saccharum based on comparative genomics. BMC Plant Biol. 2018, 18, 270. [CrossRef] [PubMed]

55. Seitz, C.; Ameres, S.; Schlangen, K.; Forkmann, G.; Halbwirth, H. Multiple evolution of flavonoid 3',5'-hydroxylase. Planta 2015, 242, 561-573. [CrossRef] [PubMed]

56. He, F.; Kang, J.; Zhou, X.; Su, Z.; Qu, L.; Gu, H. Variation at the transcriptional level among Chinese natural populations of Arabidopsis thaliana in response to cold stress. Chin. Sci. Bull. 2008, 53, 2989-2999. [CrossRef]

57. Wei, X.; Liu, F.; Chen, C.; Ma, F.; Li, M. The Malus domestica sugar transporter gene family: Identifications based on genome and expression profiling related to the accumulation of fruit sugars. Front. Plant Sci. 2014, 5, 569. [CrossRef]

58. Laby, R.J.; Kincaid, M.S.; Kim, D.; Gibson, S.I. The Arabidopsis sugar-insensitive mutants sis4 and sis5 are defective in abscisic acid synthesis and response. Plant J. 2000, 23, 587-596. [CrossRef]

59. Arenas-Huertero, F.; Arroyo, A.; Zhou, L.; Sheen, J.; León, P. Analysis of Arabidopsis glucose insensitive mutants, gin5 and gin6, reveals a central role of the plant hormone ABA in the regulation of plant vegetative development by sugar. Genes Dev. 2000, 14, 2085-2096. [CrossRef]

60. Cheng, W.-H.; Endo, A.; Zhou, L.; Penney, J.; Chen, H.-C.; Arroyo, A.; Leon, P.; Nambara, E.; Asami, T.; Seo, M.; et al. A Unique Short-Chain Dehydrogenase/Reductase in Arabidopsis Glucose Signaling and Abscisic Acid Biosynthesis and Functions. Plant Cell 2002, 14, 2723-2743. [CrossRef]

61. Beyer, E.M.; Morgan, P.W. Abscission: The role of ethylene modification of auxin transport. Plant Physiol. 1971, 48, 208-212. [CrossRef] [PubMed]

62. León, P.; Sheen, J. Sugar and hormone connections. Trends Plant. Sci. 2003, 8, 110-116. [CrossRef]

63. Ru, L.; He, Y.; Zhu, Z.; Patrick, J.W.; Ruan, Y.-L. Integrating Sugar Metabolism With Transport: Elevation of Endogenous Cell Wall Invertase Activity Up-Regulates SIHT2 and SISWEET12c Expression for Early Fruit Development in Tomato. Front. Genet. 2020, 11, 1260. [CrossRef] [PubMed]

64. Zhang, X.; Feng, C.; Wang, M.; Li, T.; Liu, X.; Jiang, J. Plasma membrane-localized SISWEET7a and SISWEET14 regulate sugar transport and storage in tomato fruits. Hortic. Res. 2021, 8, 186. [CrossRef] [PubMed]

65. Ho, L.-H.; Klemens, P.A.W.; Neuhaus, H.E.; Ko, H.-Y.; Hsieh, S.-Y.; Guo, W.-J. SISWEET1a is involved in glucose import to young leaves in tomato plants. J. Exp. Bot. 2019, 70, 3241-3254. [CrossRef]

66. Heineke, D.; Wildenberger, K.; Sonnewald, U.; Willmitzer, L.; Heldt, H.W. Accumulation of hexoses in leaf vacuoles: Studies with transgenic tobacco plants expressing yeast-derived invertase in the cytosol, vacuole or apoplasm. Planta 1994, 194, 29-33. [CrossRef]

67. Fan, C.; Feng, S.; Huang, J.; Wang, Y.; Wu, L.; Li, X.; Wang, L.; Tu, Y.; Xia, T.; Li, J. AtCesA8-driven OsSUS3 expression leads to largely enhanced biomass saccharification and lodging resistance by distinctively altering lignocellulose features in rice. Biotechnol. Biofuels 2017, 10, 221. [CrossRef]

68. Nguyen, Q.A.; Luan, S.; Wi, S.G.; Bae, H.; Lee, D.-S.; Bae, H.-J. Pronounced Phenotypic Changes in Transgenic Tobacco Plants Overexpressing Sucrose Synthase May Reveal a Novel Sugar Signaling Pathway. Front. Plant Sci. 2016, 6, 1216. [CrossRef]

69. Hockema, B.; Etxeberria, E. Metabolic Contributors to Drought-enhanced Accumulation of Sugars and Acids in Oranges. J. Am. Soc. Hortic. Sci. 2001, 126, 599-605. [CrossRef]

70. Stitt, M. Pyrophosphate as an Energy Donor in the Cytosol of Plant Cells: An Enigmatic Alternative to ATP. Bot. Acta 1998, 111, 167-175. [CrossRef]

71. Bologa, K.L.; Fernie, A.R.; Leisse, A.; Ehlers Loureiro, M.; Geigenberger, P. A Bypass of Sucrose Synthase Leads to Low Internal Oxygen and Impaired Metabolic Performance in Growing Potato Tubers. Plant Physiol. 2003, 132, 2058-2072. [CrossRef] [PubMed]

72. Katoh, K.; Standley, D.M. A simple method to control over-alignment in the MAFFT multiple sequence alignment program. Bioinformatics 2016, 32, 1933-1942. [CrossRef] [PubMed]

73. Mikita, S.; David, T.; Peer, B. PAL2NAL: Robust conversion of protein sequence alignments into the corresponding codon alignments. Nucleic Acids Res. 2006, 34, W609-W612.

74. Lam-Tung, N.; Schmidt, H.A.; Arndt, V.H.; Quang, M.B. IQ-TREE: A Fast and Effective Stochastic Algorithm for Estimating Maximum-Likelihood Phylogenies. Mol. Biol. Evol. 2015, 32, 268-274.

75. Hu, K. Become Competent in Generating RNA-Seq Heat Maps in One Day for Novices Without Prior R Experience. Methods Mol. Biol. 2021, 2239, 269-303. [CrossRef] [PubMed]

76. Tang, Y.; Ren, J.; Liu, C.; Jiang, J.; Yang, H.; Li, J. Genetic characteristics and QTL analysis of the soluble sugar content in ripe tomato fruits. Sci. Hortic. 2021, 276, 109785. [CrossRef] 